\title{
Neuronal Store-Operated Calcium Entry and Mushroom Spine Loss in Amyloid Precursor Protein Knock-In Mouse Model of Alzheimer's Disease
}

\author{
Hua Zhang, ${ }^{1}$ Lili Wu, ${ }^{1}$ Ekaterina Pchitskaya, ${ }^{2}$ OOlga Zakharova, ${ }^{2}$ Takashi Saito, ${ }^{3,4}$ Takaomi Saido, ${ }^{3}$ \\ and Ilya Bezprozvanny ${ }^{1,2}$ \\ ${ }^{1}$ Department of Physiology, University of Texas Southwestern Medical Center, Dallas, Texas 75390, 22Laboratory of Molecular Neurodegeneration, St. \\ Petersburg State Polytechnical University, St. Petersburg 195251, Russia, ${ }^{3}$ Laboratory for Proteolytic Neuroscience, RIKEN Brain Science Institute, \\ 351-0198 Saitama, Japan, and ${ }^{4}$ Japan Science and Technology Agency, 332-0012 Saitama, Japan
}

\begin{abstract}
Alzheimer's disease $(\mathrm{AD})$ is the most common reason for elderly dementia in the world. We proposed that memory loss in $\mathrm{AD}$ is related to destabilization of mushroom postsynaptic spines involved in long-term memory storage. We demonstrated previously that stromal interaction molecule 2 (STIM2)-regulated neuronal store-operated calcium entry (nSOC) in postsynaptic spines play a key role in stability of mushroom spines by maintaining activity of synaptic $\mathrm{Ca}^{2+} /$ calmodulin kinase II (CaMKII). Furthermore, we demonstrated previously that the STIM2-nSOC-CaMKII pathway is downregulated in presenilin 1 M146V knock-in (PS1-M146V KI) mouse model of AD, leading to loss of hippocampal mushroom spines in this model. In the present study, we demonstrate that hippocampal mushroom postsynaptic spines are also lost in amyloid precursor protein knock-in (APPKI) mouse model of AD. We demonstrated that loss of mushroom spines occurs as a result of accumulation of extracellular $\beta$-amyloid 42 in APPKI culture media. Our results indicate that extracellular A $\beta_{42}$ acts by overactivating mGluR 5 receptor in APPKI neurons, leading to elevated $\mathrm{Ca}^{2+}$ levels in endoplasmic reticulum, compensatory downregulation of STIM2 expression, impaired synaptic nSOC, and reduced CaMKII activity. Pharmacological inhibition of mGluR5 or overexpression of STIM2 rescued synaptic nSOC and prevented mushroom spine loss in APPKI hippocampal neurons. Our results indicate that downregulation of synaptic STIM2-nSOC-CaMKII pathway causes loss of mushroom synaptic spines in both presenilin and APPKI mouse models of AD. We propose that modulators/activators of this pathway may have a potential therapeutic value for treatment of memory loss in $\mathrm{AD}$.
\end{abstract}

Key words: calcium; imaging; synaptic; transgenic

\section{Significance Statement}

A direct connection between amyloid-induced synaptic mushroom spine loss and neuronal store-operated calcium entry pathway is shown. These results provide strong support for the calcium hypothesis of neurodegeneration and further validate the synaptic store-operated calcium entry pathway as a potential therapeutic target for Alzheimer's disease.

\section{Introduction}

Alzheimer's disease (AD) is the most common reason for elderly dementia in the world, but the causes for $\mathrm{AD}$ remain poorly

Received March 16, 2015; revised July 15, 2015; accepted July 17, 2015.

Author contributions: H.Z. and I.B. designed research; H.Z., L.W., E.P., and 0.Z. performed research; T. Saito and T. Saido contributed unpublished reagents/analytic tools; H.Z., L.W., E.P., O.Z., and I.B. analyzed data; H.Z. and I.B. wrote the paper.

This work was supported by National Institutes of Health Grant R01NS080152 (I.B.; data on Figs. 1-7) and Russian Science Foundation Grant 14-25-00024 (I.B.; data on Fig 8). I.B. is a holder of the Carl J. and Hortense M. Thomsen Chair in Alzheimer's Disease Research. We are grateful to members of the llya Bezprozvanny laboratory for advice and suggestions, Leah Taylor and Polina Plotnikova for administrative assistance, and Jianfei Lin for advice with statistical analysis procedures. We thank Pfizer for providing a sample of PF-5212391. We thank University of lowa vector core for help with production of AAV-STIM2 and AAV-GFP viruses. understood and effective therapy for AD has not yet been developed. Memory loss in AD results from "synaptic failure" (Selkoe, 2002; Koffie et al., 2011; Tu et al., 2014). There are three morphological groups of dendritic spines: mushroom, stubby, and thin spines (Kasai et al., 2003; Bourne and Harris, 2008). It has been proposed that mushroom spines are stable "memory spines" that make functionally stronger synapses and therefore responsible

I.B. is a paid consultant to Ataxion and TEVA in the field of neurodegeneration. The other authors declare no competing financial interests.

Correspondence should be addressed to Dr. llya Bezprozvanny, 5323 Harry Hines Boulevard, ND12.200, Dallas, TX 75390. E-mail: Ilya.Bezprozvanny@UTSouthwestern.edu.

DOI:10.1523/JNEUROSCI.1034-15.2015

Copyright $\odot 2015$ the authors $\quad 0270-6474 / 15 / 3513275-12 \$ 15.00 / 0$ 
for memory storage (Bourne and Harris, 2007). We and others proposed previously that mushroom spines are eliminated strongly in $\mathrm{AD}$ and that loss of mushroom spines may underlie cognitive decline during the progression of the disease (Tackenberg et al., 2009; Popugaeva et al., 2012; Popugaeva and Bezprozvanny, 2013). However, cell biological mechanisms responsible for loss of mushroom spines in $\mathrm{AD}$ are poorly understood.

In a recent study, we demonstrated that neuronal storeoperated calcium entry (nSOC) in postsynaptic spines play a key role in stability of mushroom spines (Sun et al., 2014). We further demonstrated that synaptic nSOC is controlled by stromal interaction molecule 2 (STIM2) and that the STIM2-nSOC pathway is downregulated in hippocampal neurons from the mutant mice containing M146V familial AD mutation in presenilin 1 (PS1M146V KI; Sun et al., 2014). Moreover, we have demonstrated that expression of STIM2 protein rescues synaptic nSOC and mushroom spine loss in PS1-M146V KI hippocampal neurons (Sun et al., 2014). Our results also indicated that impairment in the STIM2-nSOC pathway may play a role in synaptic loss in sporadic AD and aging (Sun et al., 2014).

Synaptotoxic effects of $\beta$-amyloid $42\left(\mathrm{~A} \beta_{42}\right)$ peptides are believed to be one of the key components of pathogenic process in AD (Koffie et al., 2011; Mucke and Selkoe, 2012; Tu et al., 2014). However, the PS1-M146V KI mice do not express human amyloid precursor protein (APP) and do not generate human $\mathrm{A} \beta_{42}$. What are the cell biological mechanisms responsible for $\mathrm{A} \beta$ induced synaptic loss? To answer this question, we investigated the importance of the STIM2-nSOC pathway in conditions of amyloid toxicity. In these experiments, we took advantage of a recently developed APP knock-in (APPKI) model of AD (Saito et al., 2014). Similar to PS1-M146V KI mice, we observed loss of mushroom spines and reduction in the STIM2-nSOC pathway in APPKI neurons. We also discovered that overexpression of STIM2 protein prevents loss of mushroom spines in APPKI neurons. Based on these results, we concluded that downregulation of the STIM2-mediated nSOC pathway is a common mechanism of synaptic loss in $\mathrm{AD}$ and that modulators/activators of this pathway may have a potential therapeutic value for treatment of memory loss in AD.

\section{Materials and Methods}

Animals. APPKI mice were described recently as APP ${ }^{\mathrm{NL}-\mathrm{F}} \mathrm{KI}$ (Saito et al., 2014). Wild-type (WT) mice of the same strain (C57BL/6) were used in control experiments. APPKIGFP mice were generated by crossing APPKI mice with line-M GFP (C57BL/6 strain) mice (Feng et al., 2000). The APPKI and APPKIGFP colonies were established and housed in a vivarium (four per cage) with a $12 \mathrm{~h}$ light/dark cycle at the University of Texas Southwestern Medical Center barrier facility. All procedures involving mice were approved by the Institutional Animal Care and Use Committee of the University of Texas Southwestern Medical Center at Dallas, in accord with the National Institutes of Health Guidelines for the Care and Use of Experimental Animals. The mice of either sex were used as a source of hippocampal neurons and brain slice experiments.

Dendritic spine analysis in primary hippocampal neural cultures. The hippocampal cultures of APPKI and WT mice were established from postnatal day $0-1$ pups and maintained in culture as we described previously (Zhang et al., 2010). For assessment of synapse morphology, hippocampal cultures were transfected with tdTomato plasmid at DIV7 using the calcium phosphate method and fixed (4\% formaldehyde and $4 \%$ sucrose in PBS, pH 7.4) at DIV15-DIV16. A Z-stack of optical section was captured using $100 \times$ objective with a confocal microscope (Carl Zeiss Axiovert 100M with LSM510). Sixteen to 20 cultured neurons from three batches of cultures were used for quantitative analysis per genotype. Quantitative analysis for dendritic spines was performed by using NeuronStudio software package (Rodriguez et al., 2008). To classify the shape of neuronal spines in culture, we adapted an algorithm from a published method (Rodriguez et al., 2008). For classification of spine shapes, we used the following cutoff values: aspect ratio for thin spines $\left(\right.$ AR_thin $\left._{\text {(crit) }}\right), 2.5$; head/neck ratio $\left(\mathrm{HNR}_{\text {(crit) }}\right), 1.4$; and head diameter $\left(\mathrm{HD}_{(\text {crit) }}\right), 0.5 \mu \mathrm{m}$. These values were defined and calculated exactly as described previously (Rodriguez et al., 2008).

Dendritic spine analysis in mice hippocampus. To analyze the shape of the spines in mice hippocampus, we used WTGFP and APPKIGFP mice. Mice of different ages and genotypes (as indicated) were perfused intracardially with ice-cold $4 \%$ paraformaldehyde (PFA) solution in $30 \mathrm{ml}$ of phosphate buffer, $\mathrm{pH} 7.4$, for $3 \mathrm{~min}$. The brains were extracted and postfixed in $4 \%$ PFA solutions for $16 \mathrm{~h}$ before cutting. Hippocampal sections $(50 \mu \mathrm{m})$ from the fixed brains were obtained using a vibratome (Leica 1200S). A Z-stack of optical section was captured using $100 \times$ objective with a confocal microscope (Carl Zeiss Axiovert 100M with LSM510). The Z interval was $0.5 \mu \mathrm{m}$. The apical dendrites of hippocampal CA1 pyramidal neurons were selected for taking images. Approximately 20-28 neurons from three to six mice of either sex were analyzed for each genotype and age sample. To classify the shape of neuronal spines in slices, we also used NeuronStudio software package and an algorithm (Rodriguez et al., 2008) with the following cutoff values:

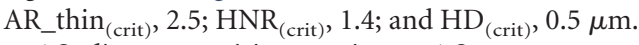

A $\beta$ oligomers toxicity experiment. $\mathrm{A} \beta_{(1-42)}$ was purchased from California Peptide. $\mathrm{A} \beta_{42}$-derived diffusible ligands (ADDLs) were prepared by following a previously published protocol (De Felice et al., 2007). Briefly, $\mathrm{A} \beta_{(1-42)}$ was prepared in aliquots as a dried hexafluoro-2propanol film and stored at $-80^{\circ} \mathrm{C}$. The peptide film was dissolved in undiluted, sterile $\mathrm{Me}_{2} \mathrm{SO}$ to make a $5 \mathrm{~mm}$ solution. The solution was diluted to $100 \mu \mathrm{M}$ with Ham's F-12 medium without glutamine (BioSource) and aged overnight at $4^{\circ} \mathrm{C}$. The preparation was centrifuged at $14,000 \times g$ for $10 \mathrm{~min}$ at $4^{\circ} \mathrm{C}$ to remove insoluble aggregates (protofibrils and fibrils). The supernatant containing soluble $\mathrm{A} \beta_{42}$ ADDL was added to DIV15 WT hippocampal culture for $48 \mathrm{~h}$, and then cells were fixed for analysis. Concentration of ADDLs was estimated based on starting amount of $\mathrm{A} \beta_{42}$ peptides.

$A \beta_{40}$ and $A \beta_{42}$ measurements in hippocampal culture medium. The hippocampal culture medium was collected at DIV15, and $\mathrm{A} \beta_{40}$ and $\mathrm{A} \beta_{42}$ concentrations were measured by Invitrogen ELISA kit (KHB3481 and KHB3441) by following the instructions of the manufacturer.

Fura- $2 \mathrm{Ca}^{2+}$ imaging experiments. Fura- $2 \mathrm{Ca}^{2+}$ imaging experiments with cultured DIV15-DIV16 hippocampal neurons were performed as described previously (Zhang et al., 2010). Fura-2 340 nm/380 nm ratio images were collected using a DeltaRAM-X illuminator, Evolve camera, and IMAGEMASTER PRO software (all from Photon Technology International). The entire cell somas were set as the region of interest (ROI) for image analysis. In ionomycin (IO) pool experiments, the cells were treated with $5 \mu \mathrm{M}$ IO addition after $30 \mathrm{~s}$ incubation in $\mathrm{Ca}^{2+}$-free media. In nSOC experiments, the neurons were moved from artificial CSF (aCSF; in mu: $140 \mathrm{NaCl}, 5 \mathrm{KCl}, 1 \mathrm{MgCl}_{2}, 2 \mathrm{CaCl}_{2}$, and 10 HEPES, pH 7.3) to calcium-free aCSF with addition of $0.4 \mathrm{~mm}$ EGTA and $1 \mu \mathrm{M}$ thapsigargin (Tg) for $30 \mathrm{~min}$ and then returned to aCSF with $1 \mu \mathrm{M}$ TTX, $50 \mu \mathrm{M}$ AP-5, $10 \mu \mathrm{M}$ CNQX, and $50 \mu \mathrm{M}$ nifedipine. The maximal amplitude (peak) of nSOC-mediated $\mathrm{Ca}^{2+}$ increase was determined from fura-2 $340 \mathrm{~nm} / 380 \mathrm{~nm}$ ratio. All $\mathrm{Ca}^{2+}$ imaging experiments were performed at room temperature.

GCamp5.3 $\mathrm{Ca}^{2+}$ imaging experiments. GCamp5.3 imaging experiments were performed as we reported previously (Sun et al., 2014). Briefly, cultured WT and KI hippocampal neurons were transfected with GCamp5.3 expression plasmid using the calcium phosphate transfection method at DIV7. The GCamp5.3 fluorescent images were collected using Olympus IX70 inverted epifluorescence microscope equipped with a $60 \times$ lens, Cascade 650 digital camera (Roper Scientific), and Prior Lumen 200 illuminator. The experiments were controlled by the MetaFluor image acquisition software package (Universal Imaging). To measure synaptic nSOC, the neurons were moved from aCSF to calcium-free media with $0.4 \mathrm{~mm}$ EGTA and $1 \mu \mathrm{M}$ Tg for $30 \mathrm{~min}$; after recording basal fluorescent signals in $\mathrm{Ca}^{2+}$-free media for $30 \mathrm{~s}, 100 \mu \mathrm{M}(R S)-3,5-$ dihydroxyphenylglycine (DHPG) in calcium-free aCSF was added and, 50 s later, the neurons were returned to aCSF with the addition of $\mathrm{Ca}^{2+}$ 
channels inhibitor mixture ( $1 \mu \mathrm{M}$ TTX, $50 \mu \mathrm{M}$ AP-5, $10 \mu \mathrm{M}$ CNQX, and $50 \mu \mathrm{M}$ nifedipine). Analysis of the data was performed using NIH ImageJ software. The ROI used in the image analysis was chosen to correspond to spines. All $\mathrm{Ca}^{2+}$ imaging experiments were done in room temperature.

Western blot analysis. DIV15 hippocampal neuron culture lysates were separated by SDS-PAGE and analyzed by Western blotting with antiSTIM1 polyclonal antibody (1:500; 4916s; Cell Signaling Technology), anti-STIM2 polyclonal antibody (1:500; 4917s; Cell Signaling Technology), anti-phospho-Ca ${ }^{2+} /$ calmodulin kinase II (CaMKII; 1:1000; 3361s; Cell Signaling Technology), anti-CaMKII (1:1000; MAB8699; Millipore Bioscience Research Reagents), anti-postsynaptic density 95 (PSD95; 1:1000; 3450s; Cell Signaling Technology), and anti-GAPDH (1:1000; MAB374; Millipore). HRP-conjugated anti-rabbit and anti-mouse secondary antibodies (115-035-146 and 111-035-144) were from Jackson ImmunoResearch. Analysis of the data from three batches of cultures was performed using Quantity One software. The mean density of each band was normalized to GAPDH signal in the same sample and averaged.

Stereotaxic injection. Preparation of AAV2/1CMVSTIM2WT-ThrGFP (AAV-STIM2) and AAV2/1CMV-NLS-GFP (AAV-GFP) viruses was described previously (Sun et al., 2014). For bilateral hippocampus stereotaxic injections, the Hamilton injection syringes were positioned at a $10^{\circ}$ angle on both sides with the following injection coordinates (from bregma): anteroposterior, $-2.0 \mathrm{~mm}$; lateral, $+2.6 \mathrm{~mm}$; and dorsoventral, $-1.9 \mathrm{~mm}$. In these experiments, $4 \mu \mathrm{l}$ of virus stocks was injected on each side. The mice were injected at 8 weeks of age and analyzed at 6 months of age. In each group, six mice of either sex were injected, and four to five neurons from each mice were analyzed for each group. In WTGFP mice injected with AAV-GFP virus, only three mice survived, so three mice were used for the analysis in this group, with seven to eight neurons from each mice analyzed.

Statistical analyses. The results are presented as mean \pm SEM. Statistical comparisons of results obtained in experiments were performed by Student's $t$ test for two-group comparisons and one-way or two-way ANOVA, followed by Tukey's test for multiple comparisons among more than two groups. The $p$ values are indicated in the text and figure legends as appropriate. The statistical analysis was performed, with $n$ indicating the number of independent neurons or spines analyzed. To validate this analysis method, a pilot statistical analysis of the data was performed using a hierarchical linear model. It was determined that mice-to-mice variability in mushroom spine fraction is only $\sim 1 / 10$ of cell-to-cell variability. Because variability between mice is not significant, we concluded that it is statistically correct to treat each cell as independent measurement and apply simple $t$ test to the analysis of mushroom spine fraction.

\section{Results}

Loss of mushroom spines in APPKI hippocampal neurons

In a previous study, we reported loss of mushroom spines in PS1-M146V KI neurons (Sun et al., 2014). To analyze the stability of synaptic spines in conditions of amyloid toxicity, we took an advantage of the recently generated APPKI model of AD (Saito et al., 2014). When compared with widely used APP transgenic mouse lines, in APPKI mice, expression of mutant APP gene [Swedish (KM670/671NL) and Beyreuther/Iberian (I716F) mutations] is under control of the endogenous promoter, resulting in physiological expression of APP and overproduction of $\mathrm{A} \beta_{42}$ and more faithful recapitulation of pathological features of human $\mathrm{AD}$, although putting the two independent FAD mutations together may potentially exert unexpected effects as has been discussed previously (Saito et al., 2014). To study the morphology of hippocampal spines in APPKI mice, we used the same approach as in our previous studies with PS1-M146V KI mice (Sun et al., 2014; Zhang et al., 2015). In these experiments, WT and APPKI primary hippocampal cultures were transfected with tdTomato plasmid, fixed at DIV15-DIV16, and analyzed by confocal imaging (Fig. 1A). The obtained confocal images were used to quantify the density and morphology of the spines by using an automated scoring procedure (Rodriguez et al., 2008). As a result of this analysis, we observed no significant difference in total spine density between WT and APPKI cultures (Fig. 1B). However, we observed a significant reduction in the fraction of the mushroom spines in the APPKI cultures. On average, the fraction of mushroom spines was equal to $27.5 \pm 1.0 \%(n=16)$ for WT cultures and $17.5 \pm 1.2 \%(n=19 ; p<0.001)$ for APPKI cultures (Fig. $1 B$ ). The fractions of stubby and thin spines was proportionally elevated in APPKI cultures (Fig. 1B).

To confirm these findings in vivo, we crossed APPKI mice with line-M GFP mice (Feng et al., 2000) to generate APPKIGFP mice. Line-M GFP mice (WTGFP) were used as a control in these experiments. We prepared hippocampal brain slices from WTGFP and APPKIGFP mice at 3 and 6 months of age and collected confocal images of the spines in the secondary apical dendrites of hippocampal CA1 neurons (Fig. 1C,E). Spine shapes and densities were determined from these images by using an automated scoring procedure (Rodriguez et al., 2008). The total spine density was similar in 3-month-old WTGFP and APPKIGFP slices (Fig. 1D). At 3 months of age, the average fraction of mushroom spines was equal to $26.0 \pm 1.5 \%(n=21)$ for WTGFP slices and $21.4 \pm 0.9 \%(n=21 ; p<0.05)$ for APPKIGFP slices (Fig. $1 D)$. The fraction of thin spines was proportionally elevated in APPKIGFP slices (Fig. 1D). The total spine density was significantly increased in 6-month-old APPKIGFP slices (Fig. $1 F$ ). The average fraction of mushroom spines increased to $35.6 \pm 1.3 \%$ $(n=25)$ in WTGFP 6-month-old slices (Fig. $1 F)$. In contrast, the average fraction of mushroom spines remained at $22.0 \pm 1.0 \%$ $(n=25 ; p<0.001$; Fig. $1 F)$ in 6-month-old APPKIGFP slices (Fig. $1 F$ ). The fractions of both stubby and thin spines were proportionally increased in 6-month-old APPKIGFP slices (Fig. $1 F$ ).

Both in vitro (Fig. $1 A, B$ ) and in vivo (Fig. $1 C-F$ ) analyses suggest that mushroom spines are lost in aging APPKI hippocampal neurons. This conclusion is similar to our previous analysis of PS1-M146V KI hippocampal neurons (Sun et al., 2014). Interestingly, in PS1-M146V KI neurons, loss of mushroom spines was compensated primarily by an increase in thin spines (Sun et al., 2014), but, in APPKI neurons, it is compensated by an increase in both thin and stubby spines (Fig. 1). Consistent with our findings with APPKI mice, fraction of mushroom spines was reduced significantly and the fraction of stubby spines was increased significantly in hippocampal slice cultures from $\mathrm{APP}_{\mathrm{SDL}}$ transgenic mice (Tackenberg and Brandt, 2009).

\section{Extracellular $\mathrm{A} \boldsymbol{\beta}_{42}$ causes mushroom spine loss in APPKI hippocampal neurons}

What causes mushroom spine loss in APPKI neurons? The APPKI neurons generate $A \beta_{42}$ inside the cells and also release it to the extracellular media. To determine whether extracellular $\mathrm{A} \beta_{42}$ can lead to loss of mushroom spines, we evaluated effects of synthetic $\mathrm{A} \beta_{42}$. In these experiments, $\mathrm{A} \beta_{42} \mathrm{ADDL}$ were prepared by following previously published protocols (De Felice et al., 2007). Hippocampal neurons from WT mice were transfected with tdTomato plasmid and challenged by 1 or $5 \mu \mathrm{M} \mathrm{A} \beta_{42}$ ADDLs at DIV15. After $48 \mathrm{~h}$ exposure to $\mathrm{A} \beta_{42}$ ADDLs, neurons were fixed and spine shapes were analyzed by confocal images (Fig. $2 A$ ). In these experiments, we discovered that application of $\mathrm{A} \beta_{42}$ ADDLs causes dose-dependent loss of mushroom spines in hippocampal neurons (Fig. $2 B$ ), consistent with the extracellular mode of action of $\mathrm{A} \beta_{42}$.

To further understand the reasons for mushroom spine loss in APPKI neurons, we quantified amounts of $\mathrm{A} \beta_{40}$ and $\mathrm{A} \beta_{42}$ in the neuronal culture media. By using ELISA, we determined that the levels of $\mathrm{A} \beta_{40}$ in the DIV15 culture media are equal to $58 \pm 10$ 
A

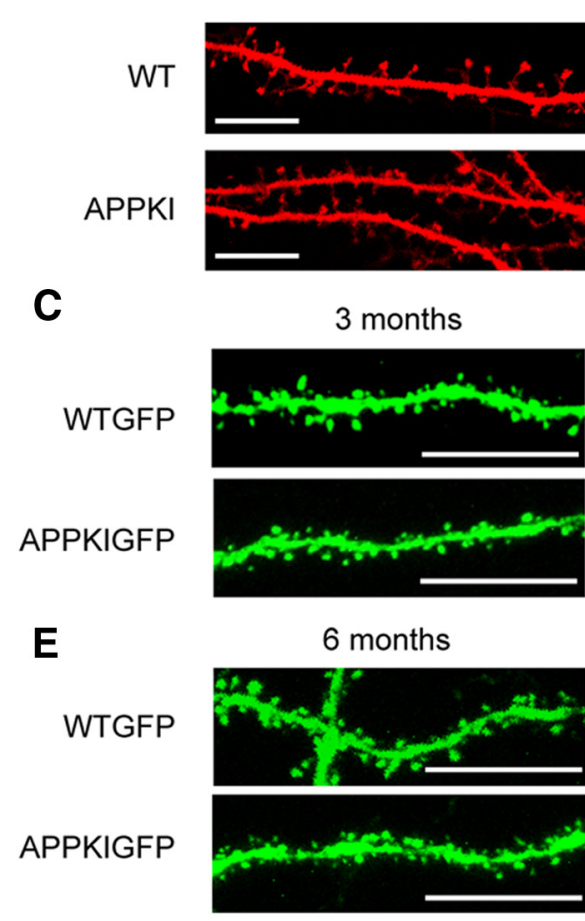

B

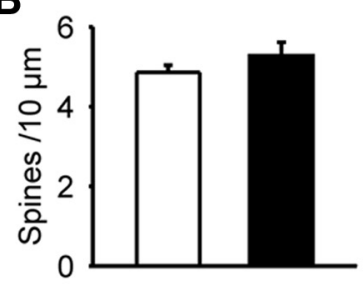

D
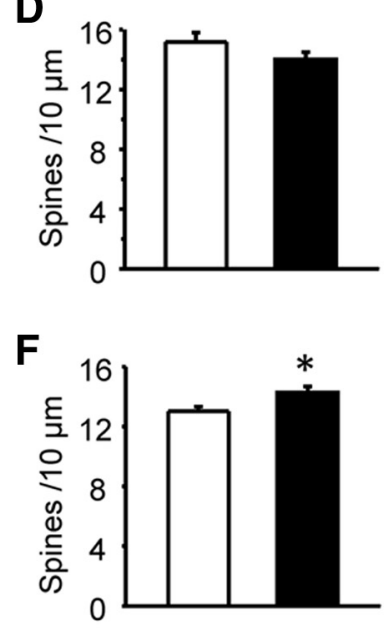

APPKI
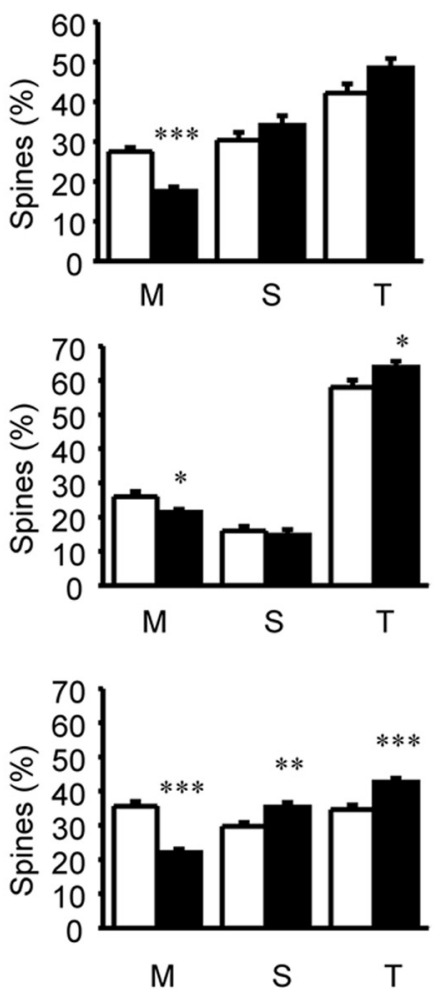

Figure 1. Loss of mushroom spines in APPKI hippocampal neurons. $A$, Confocal images of WT or APPKI DIV15 primary hippocampal neurons transfected with tdTomato. Scale bars, $10 \mu \mathrm{m}$. $\boldsymbol{B}$, Total spine density and a fraction of various spine types (M, mushroom; $S$, stubby; $T$, thin) in hippocampal neuronal cultures from DIV15 WT and APPKI mice. The data are shown as mean $\pm S E$ ( $n \geq 16$ neurons from 3 batches of cultures). $\boldsymbol{C}, \boldsymbol{E}$, Confocal images of CA1 hippocampal neurons from 3-month-old ( $\boldsymbol{C}$ ) and 6-month-old $(\boldsymbol{E})$ WTGFP and APPKIGFP mice. Scale bars, $10 \mu \mathrm{m}$. $\boldsymbol{D}, \boldsymbol{F}$, Total spine density and a fraction of various spine types ( $M$, mushroom; $S$, stubby; $T$, thin) in hippocampal neurons from 3-month-old $(\boldsymbol{D})$ and 6 -month-old $(\boldsymbol{F})$ WTGFP and APPKIGFP mice. The data are shown as mean $\pm \operatorname{SE}\left(n \geq 21\right.$ neurons, 3 month data from 3 mice of each group, 6 month data from 5 mice of each group). ${ }^{*} p<0.05,{ }^{* *} p<0.01,{ }^{* * *} p<0.001$.

$\mathrm{pg} / \mathrm{ml}(n=2)$, and those of $\mathrm{A} \beta_{42}$ are equal to $1657 \pm 158 \mathrm{pg} / \mathrm{ml}$ $(n=2$; Fig. $2 C)$. These results are consistent with the previous description of APPKI mice (Saito et al., 2014). In contrast to APPKI cultures, the levels of $\mathrm{A} \beta_{42}$ in WT DIV15 media are equal to only $70 \pm 30 \mathrm{pg} / \mathrm{ml}(n=2$; Fig. $2 C)$. To discriminate between intracellular and extracellular actions of $\mathrm{A} \beta_{42}$, we exchanged the media between WT and APPKI neurons on a daily basis starting at DIV7. In ELISA experiments, we determined that, after $8 \mathrm{~d}$ of media exchange (by DIV15), the levels of $\mathrm{A} \beta_{42}$ were equal to $987 \pm 143 \mathrm{pg} / \mathrm{ml}(n=2)$ for the WT cultures with APPKI media $(\mathrm{WT}+\mathrm{APPm})$ and $175 \pm 12 \mathrm{pg} / \mathrm{ml}(n=2)$ for the APPKI cultures with WT media (APP + WTm; Fig. 2C).

Hippocampal neurons from different groups were transfected with tdTomato plasmid, fixed at DIV15, and analyzed by confocal microscopy (Fig. 2D). Consistent with previous results (Fig. $1 A$ ), we determined that the fraction of mushroom spines in these experiments was equal to $32 \pm 1 \%(n=20)$ for WT cultures and $17.2 \pm 1.2 \%(n=19 ; p<0.001)$ for APPKI cultures (Fig. $2 E)$. The fraction of mushroom spines in WT + APPm was reduced to $20 \pm 1 \%(n=20 ; p<0.001$; Fig. $2 E)$. In contrast, the fraction of mushroom spines in APP + WTm was equal to $35 \pm 2 \%(n=$ 20), not significantly different from WT cultures (Fig. 2E). These results suggested that the accumulation of extracellular $\mathrm{A} \beta_{42}$ is both necessary and sufficient to induce mushroom spine loss in cultured hippocampal neurons.
Impairment of the STIM2-nSOC-CaMKII pathway in APPKI hippocampal spines

In our recent studies, we discovered that nSOC plays a key role in supporting stability of mushroom postsynaptic spines in hippocampal neurons (Sun et al., 2014). In the same study, we demonstrated that synaptic nSOC is controlled by STIM2 and that the STIM2-nSOC pathway is downregulated in PS1-M146V KI hippocampal neurons (Sun et al., 2014). Does loss of mushroom spines in APPKI hippocampal neurons also involve impairment of the STIM2-nSOC pathway? Endoplasmic reticulum (ER) $\mathrm{Ca}^{2+}$ levels play a key role in control of SOC pathway activity. In initial series of experiments, we evaluated ER $\mathrm{Ca}^{2+}$ levels in APPKI neurons. In these experiments, DIV15-DIV16 APPKI and WT hippocampal neuronal cultures were loaded with fura-2 $\mathrm{Ca}^{2+}$ imaging dye, transferred to $\mathrm{Ca}^{2+}$-free media for $30 \mathrm{~s}$, and challenged with $\mathrm{Ca}^{2+}$ ionophore IO. Application of IO resulted in $\mathrm{Ca}^{2+}$ release from the ER stores in WT and APPKI neurons (Fig. $3 A$ ). By integrating the area under the fura- 2 signal curve, we estimated that the average IO-sensitive $\mathrm{Ca}^{2+}$ pool size was equal to $7.6 \pm 0.5$ A.U. $(n=105)$ in WT neurons and $10.3 \pm 0.9$ A.U. $(n=88 ; p<0.01)$ in APPKI neurons (Fig. $3 A)$. Thus, ER Ca ${ }^{2+}$ levels appear to be elevated significantly in APPKI neurons. In the next series of experiments, we evaluated nSOC in soma of WT and APPKI neurons. In these experiments, DIV15-DIV16 APPKI and WT hippocampal neuronal cultures were loaded with fura-2 
A Ctrl

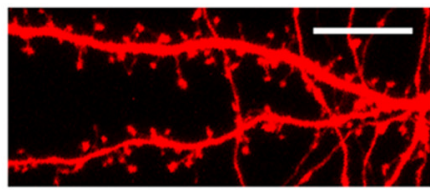

ADDL $1 \mu \mathrm{M}$

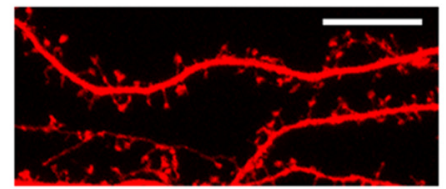

ADDL $5 \mu \mathrm{M}$

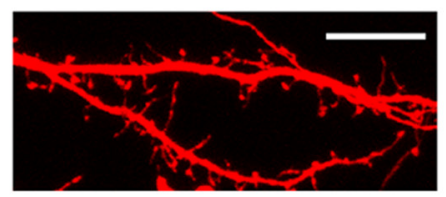

B
C

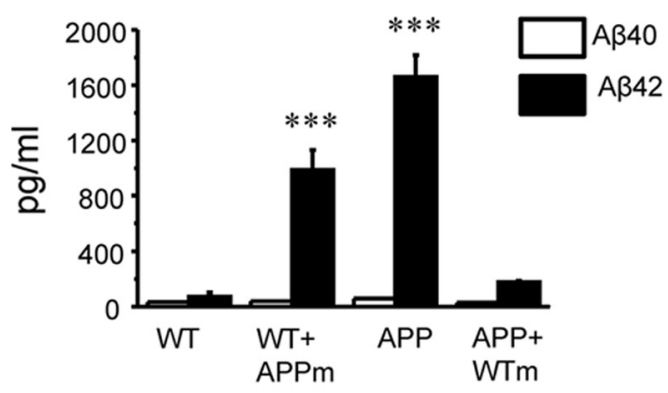

D

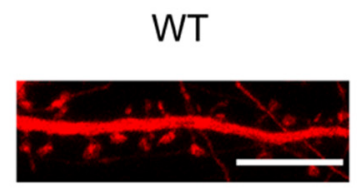

WT + APPm

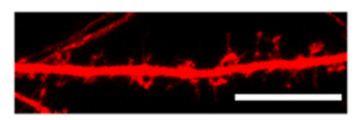

E

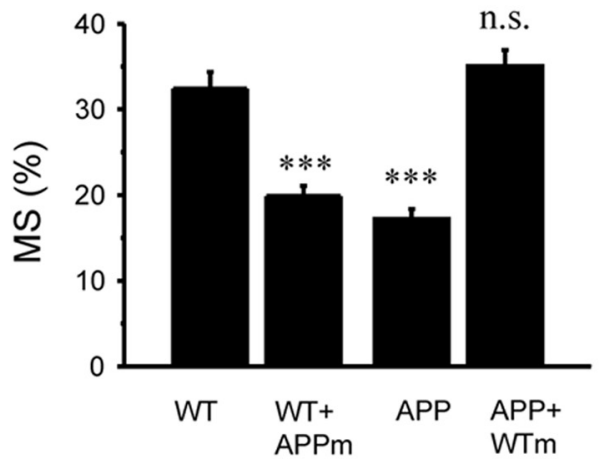

Figure 2. Extracellular $A \beta_{42}$ causes mushroom spine loss in APPKI neurons. $A$, Confocal images of WT DIV17 primary hippocampal neurons transfected with tdTomato. Neurons were exposed to 1 or $5 \mu \mathrm{m} \mathrm{A} \beta_{42}$ ADDLs for $48 \mathrm{~h}$ as indicated. Ctrl, Control. Scale bars, $10 \mu \mathrm{m}$. B, An average fraction of mushroom spines (MS) is shown for DIV17 WT neurons in control conditions and after ADDL exposure as mean $\pm \mathrm{SE}\left(n=20\right.$ neurons from 2 batches of cultures). ${ }^{* *} p<0.01,{ }^{* *} p<0.001 . C, A \beta_{40}$ and $A \beta_{42}$ concentrations in the DIV15 culture medium for WT and APPKI cultures, for WT + APPm, and for APP + WTm. The data are shown as mean \pm SE ( $n=2$ independent experiments). $D$, Confocal images of DIV15 WT, APPKI, WT + APPm, and APPKI + WTm primary hippocampal neurons transfected with tdTomato. Scale bars, $10 \mu \mathrm{m}$. $\boldsymbol{E}$, An average fraction of mushroom spines (MS) is shown for DIV15 WT, APPKI, WT + APPm, and APPKI + WTm as mean \pm SE $(n \geq 19$ neurons from 2 independent experiments). ${ }^{* *} p<0.01,{ }^{* * *} p<0.001$.

and transferred to $\mathrm{Ca}^{2+}$-free media containing $1 \mu \mathrm{M}$ sarco/endoplasmic reticulum $\mathrm{Ca}^{2+}$-ATPase pump inhibitor Tg. After depletion of $\mathrm{ER} \mathrm{Ca}^{2+}$ stores, the cultures were transferred to the media containing $2 \mathrm{mM} \mathrm{Ca}^{2+}$, and fura-2 signals representing nSOC $\mathrm{Ca}^{2+}$ influx in the soma were recorded in WT and APPKI neurons (Fig. $3 B$ ). The average peak of somatic nSOC signal was equal to $0.70 \pm 0.02(n=164)$ for WT neurons and $0.45 \pm 0.03$ $(n=129 ; p<0.001)$ for APPKI neurons (Fig. $3 B)$.

To evaluate the spine nSOC, we performed $\mathrm{Ca}^{2+}$ imaging experiments with the genetically encoded $\mathrm{Ca}^{2+}$ indicator GCamp5.3 (Tian et al., 2009). In these experiments, WT and APPKI hippocampal neurons were transfected with GCamp5.3 expression plasmid at DIV7, and $\mathrm{Ca}^{2+}$ imaging experiments were performed at DIV15-DIV16. GCamp5.3 fluorescence signal was used to visualize spine structures and to image changes in spine $\mathrm{Ca}^{2+}$ concentration, as described previously in our studies with PS1-M146V KI neurons (Sun et al., 2014). ER Ca ${ }^{2+}$ stores in the spines in these experiments were depleted by incubating GCamp5.3-transfected neurons in $\mathrm{Ca}^{2+}$-free media for $30 \mathrm{~min}$ in the presence of $1 \mu \mathrm{M}$ Tg, followed by addition of $100 \mu \mathrm{M}$ DHPG.
After store depletion, neurons were returned to the media containing $2 \mathrm{mM} \mathrm{Ca}^{2+}$, and spine nSOC signals were recorded as changes in GCamp5.3 fluorescence. Consistent with previous results (Sun et al., 2014), we observed robust nSOC signal in WT spines (Fig. 3C). In contrast, spine nSOC signal was almost completely abolished in APPKI spines (Fig. 3C). On average, the size of synaptic nSOC peak was equal to $4.2 \pm 0.4(n=250)$ in WT neurons but only $0.5 \pm 0.1(n=260 ; p<0.001)$ in APPKI neurons (Fig. 3C).

In previous studies, we discovered that the reduction in spine nSOC in PS1-M146V KI neurons can be explained by reduction in expression levels of STIM2 protein (Sun et al., 2014). To determine the reasons for impaired spine nSOC in APPKI neurons, we performed a series of Western blotting experiments with lysates from DIV15 WT and APPKI cultures. In these experiments, we found that the levels of STIM2 protein were reduced by $35 \%$ in APPKI cultures, but the levels of STIM1 protein were not significantly different (Fig. $3 D, E$ ). These results are consistent with PS1-M146V KI data (Sun et al., 2014), although STIM2 reduction in PS1-M146V KI neurons was more dramatic than in 
A
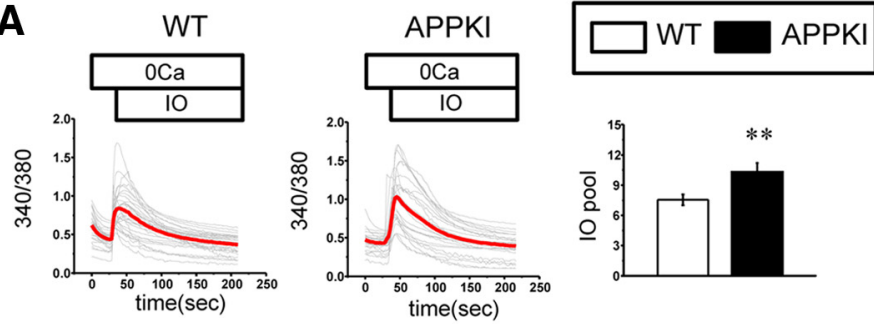

B

WT

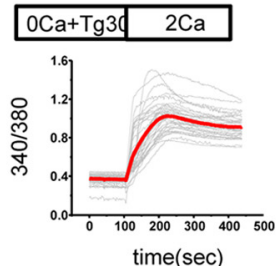

C

WT

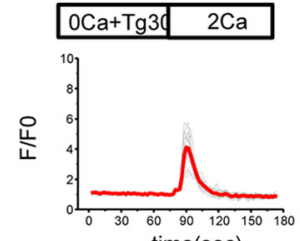

time(sec)
APPKI
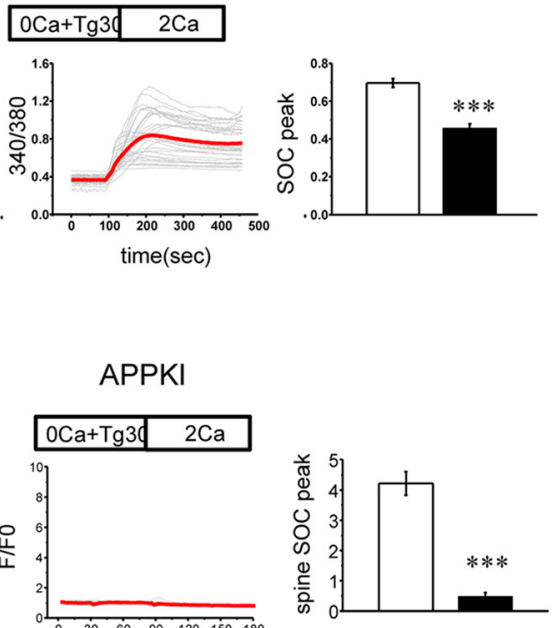

D

STIM2

STIM1

PSD95

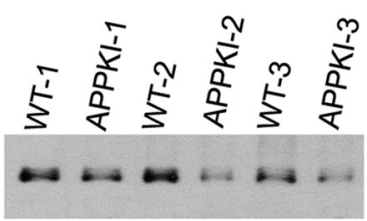

P-CAMKII

CAMKII

GAPDH
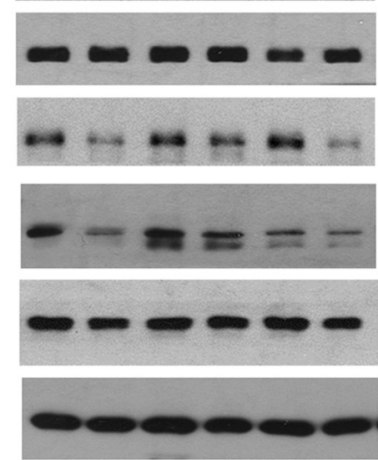

E

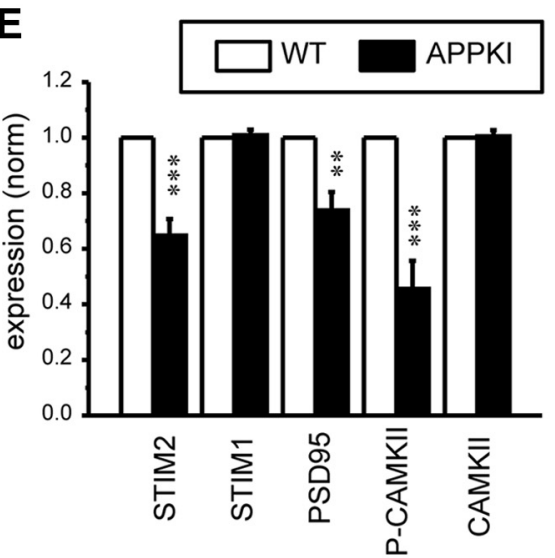

Figure 3. ER $\mathrm{Ca}^{2+}$ signaling dysregulation in APPKI hippocampal neurons. $A$, Somatic fura- $2 \mathrm{Ca}^{2+}$ signals $\left(F_{340} / F_{380}\right)$ is shown for DIV15 WT and APPKI hippocampal neurons. The neurons were incubated for $30 \mathrm{~s} \mathrm{in} \mathrm{Ca}^{2+}$-free media and challenged with $5 \mu \mathrm{m}$ I0. Individual cell traces (gray) and average traces (red) are shown for each experimental group. The I0-releasable ER Ca ${ }^{2+}$ pool was calculated as the area under 10 -induced fura- 2 signal for each neuron. The responses were averaged across different neurons and experiments and presented as mean \pm SE $(n \geq 88$ neurons from 3 batches of cultures). ${ }^{* *} p<0.01$. B, Somatic fura- $2 \mathrm{Ca}^{2+}$ signals $\left(F_{340} / F_{380}\right)$ are shown for DIV15 WT and APPKI hippocampal neurons. The neurons were incubated for 30 min in $\mathrm{Ca}^{2+}$-free media in the presence of $1 \mu \mathrm{m} \mathrm{Tg}$ and returned to the media containing $2 \mathrm{~mm} \mathrm{Ca}^{2+}$ to trigger $\mathrm{nSOC}$. Individual cell traces (gray) and average traces (red) are shown for each experimental group. The amplitude of somatic $n S O C$ was determined for each neuron. The average somatic $n S O C$ peak amplitude is shown as mean $\pm S E\left(n \geq 129\right.$ neurons from 3 batches of cultures). ${ }^{* * *} p<0.001$. $C$, Synaptic GCaMP5.3 $\mathrm{Ca}^{2+}$ signals $\left(F / F_{0}\right)$ are shown for DIV15 WT and APPKI hippocampal neurons. The neurons were incubated for 30 min in $\mathrm{Ca}^{2+}$-free media in the presence of $1 \mu \mathrm{M} \mathrm{Tg} \mathrm{and} \mathrm{returned}$ to the media containing $2 \mathrm{mM} \mathrm{Ca}^{2+}$ to trigger synaptic nSOC. The neurons were challenged by $100 \mu \mathrm{M} \mathrm{DHPG} 50$ s before addition of $2 \mathrm{~mm} \mathrm{Ca}{ }^{2+}$. The average synaptic nSOC peak responses is shown as mean \pm SE ( $n \geq 250$ spines from 6 batches of cultures). ${ }^{* * *} p<0.001$. D, The expression levels of STIM1, STIM2, PSD95, pCAMKII, and CAMKII proteins were analyzed by Western blotting of lysates from three different batches of WT and APPKI DIV15 hippocampal cultures. GAPDH was used as a loading control. $\boldsymbol{E}$, Expression levels of each protein were normalized to GAPDH for every sample. These values were normalized to WT for every batch of cells. The average values are shown as mean \pm SE ( $n=3$ batched of cultures). ${ }^{* *} p<0.01,{ }^{* * *} p<0.001$.

APPKI neurons. The levels of STIM1 were not affected in either PS1-M146V KI and APPKI cultures. Loss of mushroom spines in PS1-M146V KI cultures correlated with the loss of PSD95 protein and with reduction in the autophosphorylated (activated) form of CaMKII (Sun et al., 2014). Similarly, we observed 26\% reduction in PSD95 levels and 54\% reduction in pCaMKII levels in APPKI cultures (Fig. 3D,E). Also similar to PS1-M146V KI neurons, total levels of CaMKII were not significantly different between WT and APPKI cultures (Fig. 3D,E). The obtained results indicated that the STIM2-nSOC-CaMKII pathway is compromised in spines of both PS1-M146V KI (Sun et al., 2014) and APPKI (Fig. 3) neurons.

\section{Overactivation of mGluR5 causes mushroom spine loss in APPKI neurons}

The reduction of spine nSOC in PS1-M146V KI neurons is attributable to impaired ER $\mathrm{Ca}^{2+}$ leak function of presenilins and resulting ER $\mathrm{Ca}^{2+}$ overload (Zhang et al., 2010; Sun et al., 2014).
What is a reason for impaired spine nSOC in APPKI neurons? A number of $\mathrm{Ca}^{2+}$ signaling pathways have been implicated in synaptotoxic effects of amyloid (Demuro et al., 2010). It has been reported that $\mathrm{A} \beta_{42}$ can affect activity of NR2B NMDARs (Li et al., 2011; Ferreira et al., 2012), L-type voltage-gated calcium channels (VDCCs) (Anekonda et al., 2011; Kim and Rhim, 2011; Wang and Mattson, 2014), and mGluR5 receptors (Renner et al., 2010; Um et al., 2013). To determine whether overactivation of any of these pathways is responsible for mushroom spine loss in APPKI neurons, we transfected WT and APPKI hippocampal neurons with tdTomato plasmid and incubated these neurons at DIV15 in the presence of $10 \mu \mathrm{M}$ ifenprodil (inhibitor of NR2B NMDA receptors), $1 \mu \mathrm{M}$ nifedipine (inhibitor of L-type $\mathrm{Ca}^{2+}$ channels), or $5 \mu \mathrm{M}$ MPEP (inhibitor of mGluR5 receptors). After overnight incubation, neurons were fixed and confocal images were obtained (Fig. 4A). Consistent with previous results (Fig. 1), we observed a significant reduction in the fraction of mushroom spines in control APPKI cultures when compared with WT cul- 
A

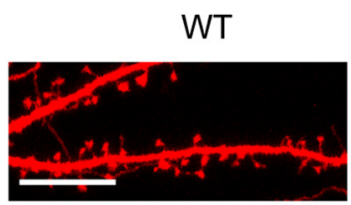

con

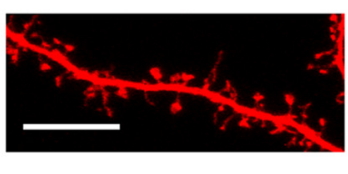

IFEN
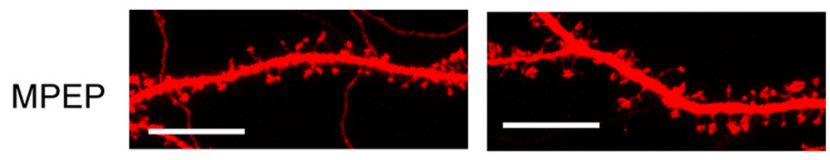

NIF
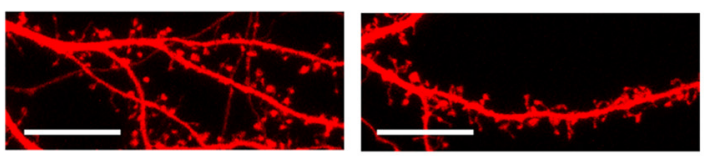

B

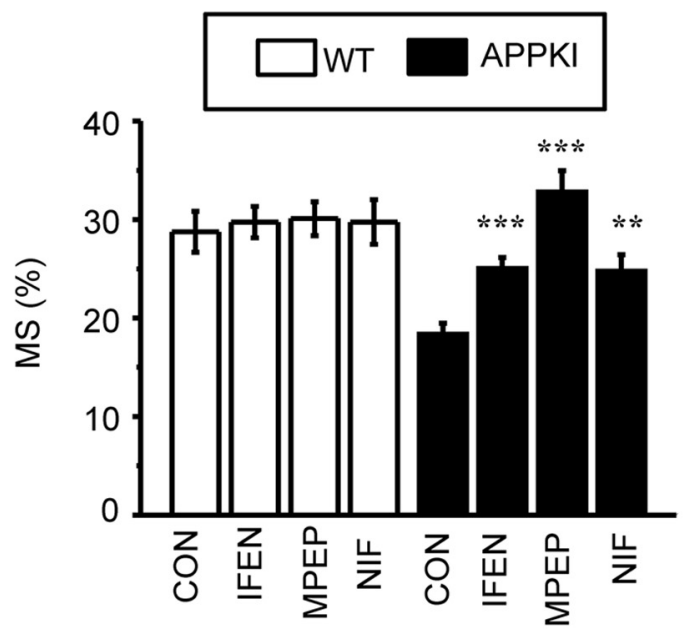

Figure 4. Evaluation of $\mathrm{Ca}^{2+}$ signaling inhibitors in mushroom spine loss assay with APPKI hippocampal cultures. A, Confocal images of DIV15 WT or APPKI DIV15 primary hippocampal neurons transfected with tdTomato. The cultures were treated for $16 \mathrm{~h}$ with $5 \mu \mathrm{m}$ ifenprodil (IFEN), $5 \mu \mathrm{m}$ MPEP, or $1 \mu \mathrm{m}$ nifedipine (NIF) before fixation. con, Control. Scale bars, $10 \mu \mathrm{m} . \boldsymbol{B}$, An average fraction of mushroom spines (MS) is shown for WT and APPKI neurons in control conditions (CON) and after exposure to $5 \mu \mathrm{m}$ ifenprodil (IFEN), $5 \mu \mathrm{M}$ MPEP, or $1 \mu \mathrm{m}$ nifedipine (NIF). At each condition, the average fraction of mushroom spines is shown as mean $\pm \mathrm{SE}(n \geq$ 20 neurons from 3 batches of cultures). ${ }^{* *} p<0.01,{ }^{* *} p<0.001$.

tures (Fig. 4B). On average, the fraction of mushroom spines in control APPKI neurons was equal to $18.3 \pm 1.1 \%(n=20)$. Incubation with the drugs had no effect on mushroom spine fraction in WT neurons (Fig. 4B). In contrast, incubation of APPKI neurons with ifenprodil resulted in an increase in mushroom spine fraction to $25.1 \pm 1 \%(n=22 ; p<0.001)$, and incubation of APPKI neurons with nifedipine resulted in an increase in mushroom spine fraction to $24.7 \pm 1.7(n=20 ; p<$ 0.01 ; Fig. $4 B$ ). Thus, inhibition of NR2B NMDARs or L-type VGCCs resulted in partial rescue of mushroom spine loss in APPKI neurons. Incubation of APPKI neurons with MPEP resulted in the most dramatic effect, resulting in an increase of mushroom spine fraction to $32.8 \pm 2.2 \%(n=20 ; p<0.001$; Fig. $4 B)$. Therefore, inhibition of mGluR5 receptors resulted in complete rescue of mushroom spine loss in APPKI neurons. In additional experiments, we confirmed these effects by using a novel specific mGluR5 antagonist, PF-5212391 [(4-(difluoromethoxy)-3- (pyridin-2-ylethynyl)phenyl) (5,7-dihydro-6H-pyrrolo[3,4-b] pyridin-6-yl)methanone, provided by Pfizer]. Similar to MPEP, overnight incubation with $1 \mu \mathrm{M}$ PF-5212391 resulted in complete rescue of mushroom spine loss in DIV15 APPKI neurons (data not shown). From these experiments, we concluded that $\mathrm{A} \beta_{42}{ }^{-}$ induced hyperactivation of mGluR5 (Renner et al., 2010; Um et al., 2013) is likely to be the main reason for mushroom spine loss in APPKI neurons.

Is there a connection between mGluR 5 overactivation and ER $\mathrm{Ca}^{2+}$ signaling dysregulation in APPKI neurons? To answer this question, we preincubated DIV15 WT and APPKI hippocampal neuronal cultures with $5 \mu \mathrm{M}$ MPEP overnight and performed fura- $2 \mathrm{Ca}^{2+}$ experiments. To measure ER $\mathrm{Ca}^{2+}$ content, WT and KI cultures were transferred to $\mathrm{Ca}^{2+}$-free media for $30 \mathrm{~s}$ and challenged with $1 \mu \mathrm{M} \mathrm{IO}$, and fura- 2 signal traces were recorded (Fig. 5A). The total size of the IO-sensitive $\mathrm{Ca}^{2+}$ pool for each group of cells was determined by integrating the area under the fura-2 signal curve. Consistent with previous findings (Fig. 3A), in these experiments, we determined that the size of the IOsensitive $\mathrm{Ca}^{2+}$ pool was $6.0 \pm 0.5(n=92)$ for WT neurons and $10.9 \pm 0.8(n=91 ; p<0.001)$ for APPKI neurons (Fig. $5 B)$. Preincubation with MPEP reduced ER $\mathrm{Ca}^{2+}$ levels in both WT and APPKI neurons. On average, after incubation with MPEP, the size of the IO-sensitive $\mathrm{Ca}^{2+}$ pool was reduced to $2.0 \pm 0.2$ $(n=101 ; p<0.001)$ in WT neurons and to $6.1 \pm 0.4(n=92 ; p<$ 0.001) in APPKI neurons (Fig. 5B).

In the next series of experiments, we measured nSOC in the soma of DIV15 WT and APPKI neurons pretreated overnight with MPEP. In these experiments, neuronal cultures were loaded with fura-2, incubated in $\mathrm{Ca}^{2+}$-free media with addition of $1 \mu \mathrm{M}$ $\mathrm{Tg}$, and moved to the media containing $2 \mathrm{mM} \mathrm{Ca}^{2+}$. The resulting fura-2 traces (Fig. $5 C$ ) were used to estimate the peak of somatic nSOC. Analysis of these experiments revealed that preincubation with MPEP had no significant effect on somatic nSOC in WT neurons (Fig. 5C,D). In contrast, preincubation with MPEP increased the peak of somatic nSOC in APPKI neurons from $0.39 \pm$ $0.02(n=77)$ to $0.63 \pm 0.02(n=106 ; p<0.001$; Fig. $5 D)$. By taking advantage of GCaMP5.3, we also performed measurements of spine nSOC in WT and APPKI neurons preincubated overnight with MPEP (Fig. 5E). In these experiments, we discovered that preincubation with MPEP had no effect on spine nSOC in WT neurons (Fig. 5E,F). In contrast, preincubation with MPEP resulted in an increase of peak spine nSOC from $0.3 \pm 0.1$ $(n=64)$ to $5.8 \pm 0.6(n=60 ; p<0.001)$ in APPKI neurons (Fig. 5E,F).

Importantly, the ER $\mathrm{Ca}^{2+}$ pool size, peak amplitude of somatic nSOC, and peak amplitude of spine nSOC in APPKI neurons pretreated overnight with MPEP were similar to the same parameters in control WT neurons (Fig. $5 B, D, F$ ). These results suggested that persistent overactivation of mGluR5 by A $\beta_{42}$ is the main reason for $\mathrm{ER} \mathrm{Ca}^{2+}$ overload and impaired somatic and synaptic nSOC in APPKI neurons.

STIM2 overexpression rescues synaptic nSOC and mushroom spine loss in APPKI hippocampal neurons

Reduction of STIM2 expression (Fig. $3 D, E$ ) is likely to be directly responsible for impaired synaptic nSOC in APPKI neurons. To test this hypothesis, we performed a series of rescue experiments. In these experiments, DIV7 WT and APPKI neurons were cotransfected with mouse STIM2 (mSTIM2) expression construct together with GCaMP5.3 imaging sensor. The spine nSOC amplitude was measured in DIV15 neuronal cultures by following the same procedures as described above. The peak synaptic 
A

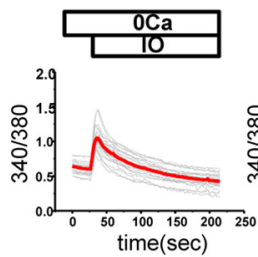

C

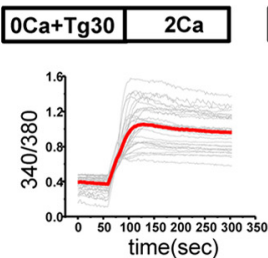

E

WT

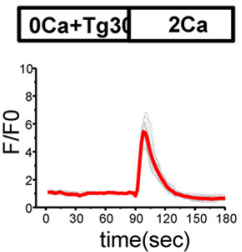

WT+MPEP

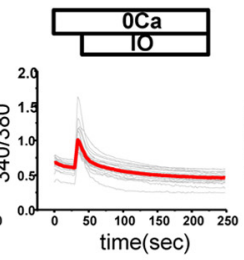

WT+MPEP

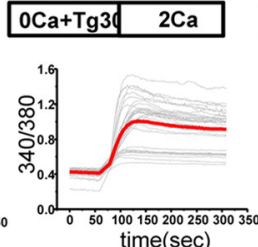

WT+MPEP

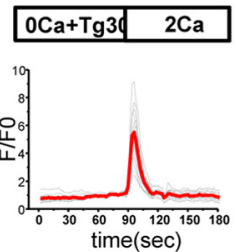

APPKI

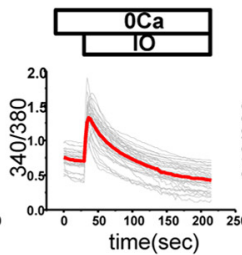

APPKI

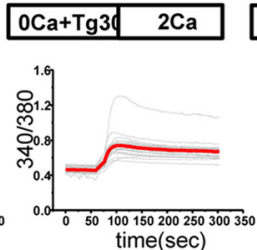

APPKI

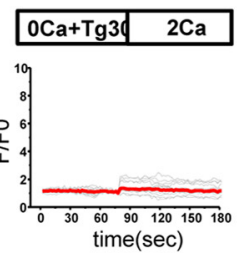

APPKI+MPEP

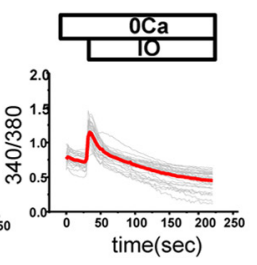

APPKI+MPEP

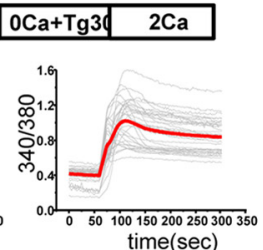

APPKI+MPEP

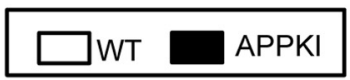

B

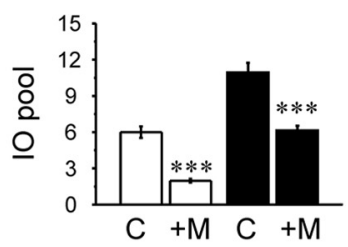

D

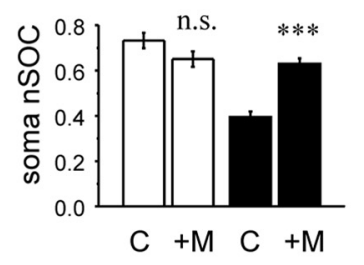

Figure 5. mGluR5 antagonist rescues $\mathrm{Ca}^{2+}$ signaling impairment in APPKI neurons. $A$, Somatic fura- $2 \mathrm{Ca}^{2+}$ signals $\left(F_{340} / F_{380}\right)$ are shown for DIV15 WT and APPKI hippocampal neurons in control conditions and after $16 \mathrm{~h}$ exposure to $5 \mu \mathrm{MMPEP}$ (+MPEP). The neurons were incubated for $30 \mathrm{sin} \mathrm{Ca}^{2+}$-free media and challenged with $5 \mu \mathrm{m} \mathrm{I0.} \mathrm{Individual} \mathrm{cell} \mathrm{traces} \mathrm{(gray)} \mathrm{and} \mathrm{average}$ traces (red) are shown for each experimental group. $\boldsymbol{B}$, The I0-releasable ER $\mathrm{Ca}^{2+}$ pool was calculated as the area under I0-induced fura-2 signal for each neuron. The responses were averaged across different neurons in each group and presented as mean $\pm S E$ ( $n \geq 91$ neurons from 3 batches of cultures). The results are compared for control group (C) and for the group after incubation with 5 $\mu \mathrm{M}$ MPEP $(+\mathrm{M}) .{ }^{* * *} p<0.001$. C, Somatic fura- $2 \mathrm{Ca}^{2+}$ signals $\left(F_{340} / F_{380}\right)$ are shown for DIV15 WT and APPKI hippocampal neurons in control conditions and after $16 \mathrm{~h}$ exposure to $5 \mu \mathrm{M}$ MPEP (+MPEP). The neurons were incubated for $30 \mathrm{~min}$ in $\mathrm{Ca}^{2+}$-free media in the presence of $1 \mu \mathrm{m} \mathrm{Tg}$ and returned to the media containing $2 \mathrm{~mm} \mathrm{Ca}^{2+}$ to trigger nSOC. Individual cell traces (gray) and average traces (red) are shown for each experimental group. $D$, The amplitude of somatic $n S O C$ was determined for each neuron. The average somatic nSOC peak amplitude is shown as mean $\pm S E$ ( $n \geq 77$ neurons from 3 batches of cultures). The results are compared for control group (C) and for the group after incubation with $5 \mu M$ MPEP $(+M)$. ${ }^{* * *} p<0.001$. E, Synaptic GCaMP5.3 Ca ${ }^{2+}$ signals $\left(F / F_{0}\right)$ are shown for DIV15 WT and APPKI hippocampal neurons in control conditions and after $16 \mathrm{~h}$ exposure to $5 \mu \mathrm{MMPEP}(+\mathrm{MPEP})$. The neurons were incubated for 30 min in $\mathrm{Ca}^{2+}$-free media in the presence of $1 \mu \mathrm{M} \mathrm{Tg}$ and returned to the media containing $2 \mathrm{~mm} \mathrm{Ca}^{2+}$ to trigger synaptic nSOC. The neurons were challenged by $100 \mu \mathrm{m} \mathrm{DHPG} 50 \mathrm{~s}$ before addition of $2 \mathrm{~mm} \mathrm{Ca}{ }^{2+} . F$, The average synaptic $n S O C$ peak responses for different groups are shown as mean $\pm S E$ ( $n \geq 60$ spines, WT groups from 4 batches of cultures, APPKI groups from 2 batches of cultures). The results are compared for the control group (C) and for the group after incubation with $5 \mu \mathrm{M} \operatorname{MPEP}(+\mathrm{M}) .{ }^{* *} p<0.001$.

nSOC in these experiments was equal to $5.3 \pm 0.6(n=146)$ in control WT neurons and $0.6 \pm 0.2(n=196)$ in control APPKI neurons (Fig. $6 A, B$ ). Expression of mSTIM2 had no significant effect on spine nSOC in WT neurons $(p>0.05$; Fig. $6 A, B)$. In contrast, expression of mSTIM2 resulted in dramatic enhancement of spine nSOC in APPKI neurons (Fig. 6A). On average, the spine nSOC amplitude in APPKI neurons transfected with mSTIM2 was equal to $5.2 \pm 0.4(n=74 ; p<0.001)$, that is, at the same level as for WT neurons (Fig. $6 B$ ).

To determine whether spine nSOC rescue is sufficient to rescue mushroom spine loss, we cotransfected WT and APPKI neurons with mSTIM2 and tdTomato expression plasmids at DIV7. The cultures were fixed at DIV15, and confocal images were obtained (Fig. 7A). Analysis of spine shapes revealed that average mushroom spine fraction in control neurons was equal to $28.5 \pm$ $1.8 \%(n=20)$ for WT neurons and $17.5 \pm 1.2 \%(n=19)$ for APPKI neurons (Fig. $7 B$ ), consistent with the previous findings (Fig. 1B). Expression of STIM2 had no effect on the fraction of mushroom spines in WT neurons but resulted in an increase in mushroom spine fraction in APPKI neurons to $35.1 \pm 1.5 \%(n=$ $20 ; p<0.001$; Fig. $7 B$ ), that is, to the same level as in WT cultures.
To further extend these findings, we infected DIV7 WT and APPKI cultures with lentiviruses encoding mSTIM2. Control cultures were infected with lentiviruses encoding nuclear targeted EGFP plasmid (GFP). The lysates from the infected cultures were collected at DIV15 and analyzed by Western blotting. As expected, infection with Lenti-mSTIM2 viruses resulted in fivefold increase in STIM2 expression levels in WT neurons and 12-fold increase in STIM2 expression levels in APPKI cultures (Fig. 7C). Importantly, we discovered that overexpression of mSTIM2 rescued PSD95 and pCaMKII levels in APPKI neurons to the same levels as in WT neurons (Fig. $7 C, D$ ). There was no effect of STIM2 expression on total CaMKII levels (Fig. 7C,D).

To validate these findings in vivo, we stereotaxically injected AAV-mSTIM2 virus (S2) to the CA1 region of 2-month-old WTGFP and APPKIGFP mice. The AAV-NLS-GFP virus (GFP) was injected in control group animals. The mice were killed at 6 months of age, and hippocampal synaptic morphology was analyzed by confocal imaging (Fig. 8A). Consistent with our previous findings (Fig. 1D), for the mice injected with AAV-NLS-GFP virus, the fraction of mushroom spines in WTGFP mice was equal to $28.0 \pm 1.2 \%(n=23)$ and in APPKIGFP mice was equal 
A
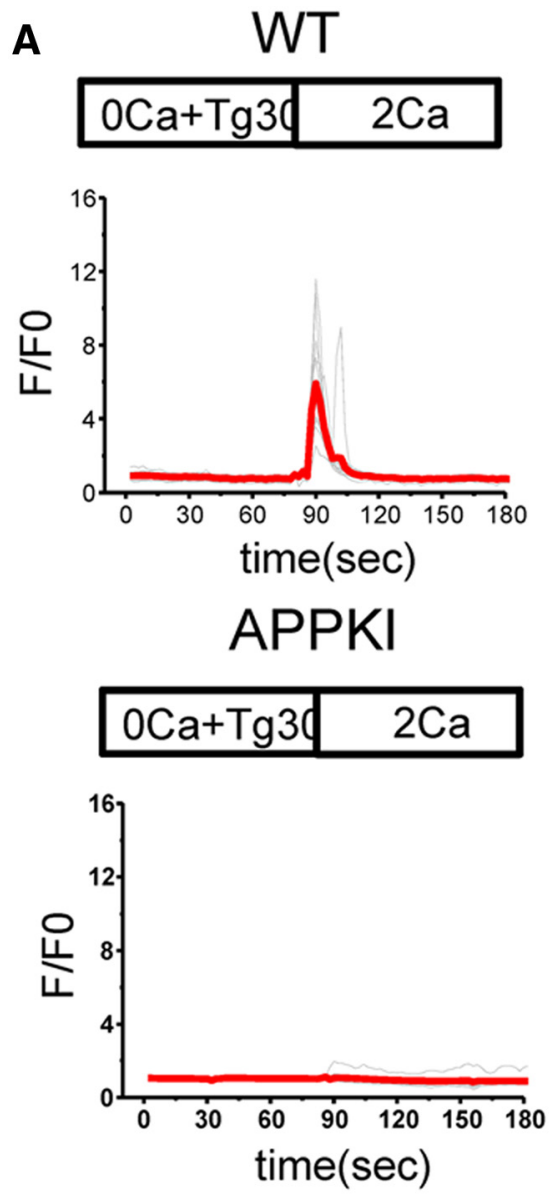

WT+STIM2
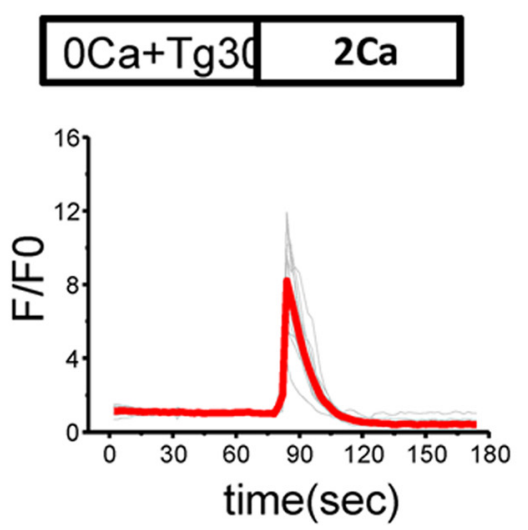

APP+STIM2
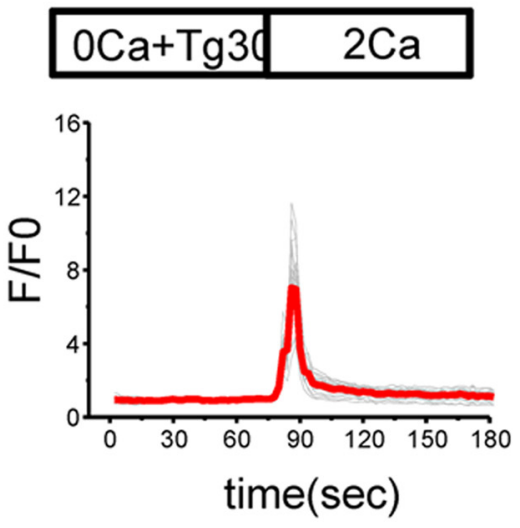

B

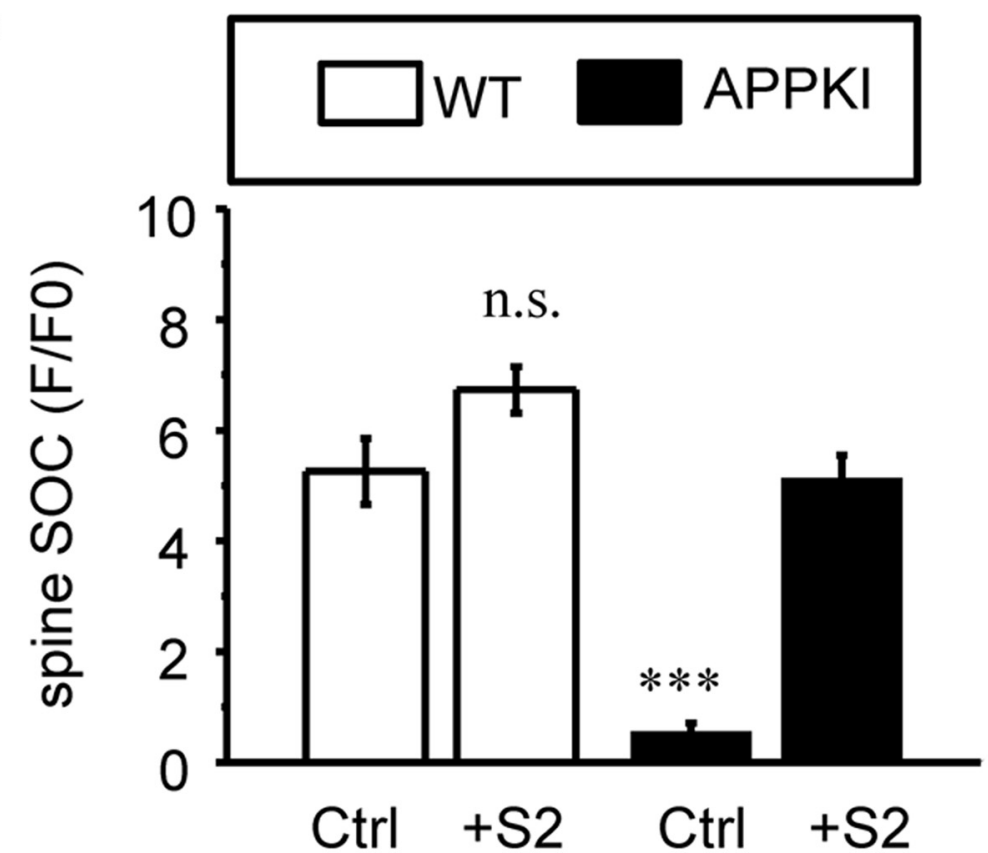

Figure 6. STIM2 overexpression rescues spine $\mathrm{nSOC}$ in APPKI neurons. $\boldsymbol{A}$, Synaptic GCaMP5.3 $\mathrm{Ca}^{2+}$ signals $\left(F / F_{0}\right)$ are shown for DIV15 WT and APPKI hippocampal neurons in control conditions and after transfection with mSTIM2 (+ STIM2). The neurons were incubated for $30 \mathrm{~min}$ in $\mathrm{Ca}^{2+}$-free media in the presence of $1 \mu \mathrm{m} \mathrm{Tg}$ and returned to the media containing $2 \mathrm{~mm} \mathrm{Ca}^{2+}$ to trigger synaptic $n S O C$. The neurons were challenged by $100 \mu \mathrm{M} \mathrm{DHPG} 50$ s before addition of $2 \mathrm{~mm}$ $\mathrm{Ca}^{2+}$. B. The average synaptic $n S O C$ peak responses for different groups are shown as mean \pm SE $(n \geq 74$ spines from 3 to 4 batches of cultures). The results are compared for control group (Ctrl) and for the group transfected with STIM2 ( + S2). ${ }^{* * *} p<0.001$.
$21.2 \pm 0.7 \%(n=26 ; p<0.001$; Fig. $8 B)$. Injection with AAV-mSTIM2 virus had no significant effect in WTGFP mice (Fig. $8 B)$. In contrast, injection with AAV-mSTIM2 virus increased the fraction of mushroom spines to $29.6 \pm 1.1 \%(n=$ 28 ) in APPKIGFP mice (Fig. $8 B$ ), that is, to the same level as in the control group of WTGFP mice.

\section{Discussion}

Loss of mushroom spines as a cell biological marker for memory loss in $\mathrm{AD}$

What is a cell biological basis for memory loss in $\mathrm{AD}$ ? It has been postulated that $\mathrm{AD}$ is a disease of synaptic failure (Selkoe, 2002; Koffie et al., 2011). Long-lasting increase in synaptic strength is a basis for persistent modification of neuronal network properties and storage of memories. It has been proposed that the mushroom spines between excitatory neurons are stable memory spines that make functionally stronger synapses and are therefore responsible for memory storage (Bourne and Harris, 2007). We and others proposed previously that loss of mushroom spines may underlie cognitive decline during the progression of $\mathrm{AD}$ (Tackenberg et al., 2009; Popugaeva et al., 2012; Popugaeva and Bezprozvanny, 2013). Recent experimental evidence in several cellular and animal models of AD provided support to this hypothesis. The fraction of mushroom spines was significantly reduced in hippocampal slice cultures from $\mathrm{APP}_{\mathrm{SDL}}$ transgenic mice (Tackenberg and Brandt, 2009). Loss of mushroom spines was observed after direct application of synthetic $\mathrm{A} \beta_{42}$ ADDLs to WT hippocampal cultures (Fig. $2 A, B$ ) or after direct injections of $\mathrm{A} \beta_{42}$ ADDLs to the hippocampus of WT mice (E. Popugaeva, E. Pchitskaya, H. Zhang, O. Vlasova, I. Bezprozvanny, unpublished observations). Anatomical analysis of spines in DU mice (APP-E693Q transgene) (Gandy et al., 2010) revealed significant shrinkage in PSD of mushroom spine synapses at 12 months of age (Price et al., 2014). In the present study, we observed a significant reduction in the fraction of mushroom spines in neuronal cultures and 6-month-old slices from the recently generated APPKI mouse model (Saito et al., 2014; Fig. 1). Loss of mushroom spines in APPKI brains (Fig. 1) occurred earlier, and it was significantly more severe than reported for DU mice (Price et al., 2014). Most likely, the concentration of $\mathrm{A} \beta_{42}$ oligomers in APPKI mice brain is significantly higher than in the DU mice, lead- 
A

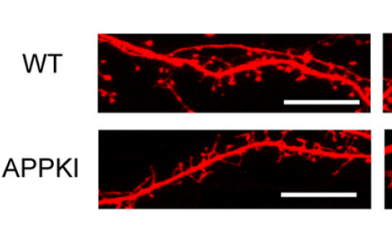

B

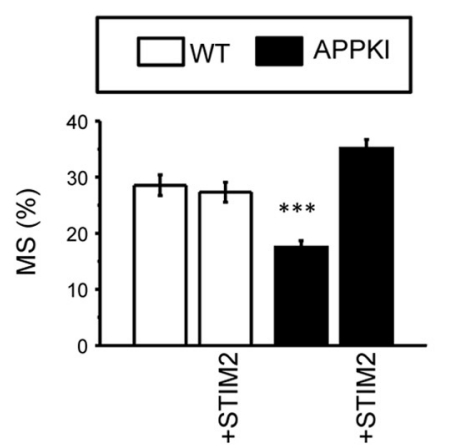

C

+ STIM2

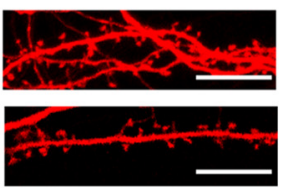

STIM2

PSD95

pCAMKII

CAMKII

GAPDH
D
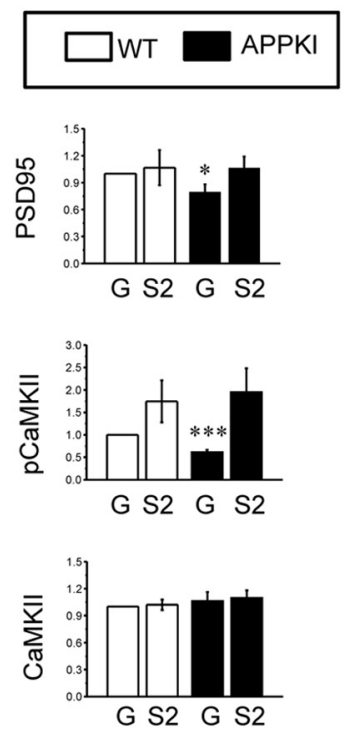

Figure 7. STIM2 overexpression rescues mushroom spines and CAMKII signaling pathway in APPKI neuronal cultures. $A$, Confocal images of WT and APPKI DIV16 primary hippocampal neurons transfected with tdTomato or cotransfected with tdTomato and mSTIM2 (+ STIM2). Scale bars, $10 \mu \mathrm{m} . \boldsymbol{B}$, An average fraction of mushroom spines (MS) is shown for DIV16 WT and APPKI neurons in control conditions and after STIM2 overexpression (+ STIM2). The data are shown as mean \pm SE ( $n \geq 19$ neurons from 3 batches of cultures). ${ }^{* * *} p<0.001$. C, The expression levels of STIM2, PSD95, pCAMKII, and CAMKII were analyzed by Western blotting of lysates from DIV15 WT and APPKI hippocampal neuronal cultures. The results with cultures infected with Lenti-NLS-GFP (G) and Lenti-mSTIM2 (S2) are compared. GAPDH was used as a loading control. $\boldsymbol{D}$, Expression levels of each protein were normalized to GAPDH for every sample. These values were normalized to Lenti-GFP-infected WT cells for every batch of cells. The average values are shown as mean \pm SE ( $n=3$ batches of cultures). ${ }^{*} p<$ $0.05,{ }^{* * *} p<0.001$.

ing to more dramatic destabilization of mushroom spines in our experiments. Interestingly, loss of mushroom spines is not unique to amyloid toxicity models. In a recent study, we observed significant loss of mushroom spines in aging hippocampal neurons from the PS1-M146V KI mouse model (Sun et al., 2014). Thus, loss of mushroom spines appears to be a common feature of several $\mathrm{AD}$ animal and cellular models, in agreement with our hypothesis that loss of mushroom spines is a relevant cell biological marker for memory loss in AD (Popugaeva et al., 2012; Popugaeva and Bezprozvanny, 2013). Notably, behavioral changes in PS1-M146V KI and APPKI mice are relatively subtle (Wang et al., 2004; Sun et al., 2005; Saito et al., 2014), suggesting that mushroom spine loss is an early event that needs to be followed by more severe neurodegenerative changes to result in stronger behavioral phenotypes.

Dysregulation of synaptic $\mathrm{Ca}^{2+}$

signaling in conditions of amyloid synaptotoxicity

What is the reason for mushroom spine loss in APPKI neurons? Conditional media exchange experiments suggested that the loss of mushroom spines in APPKI cultures is triggered by extracellular actions of $\mathrm{A} \beta_{42}$ (Fig. 2C-E). A similar conclusion was reached in experiments with direct application of synthetic $\mathrm{A} \beta_{42} \mathrm{AD}$ -
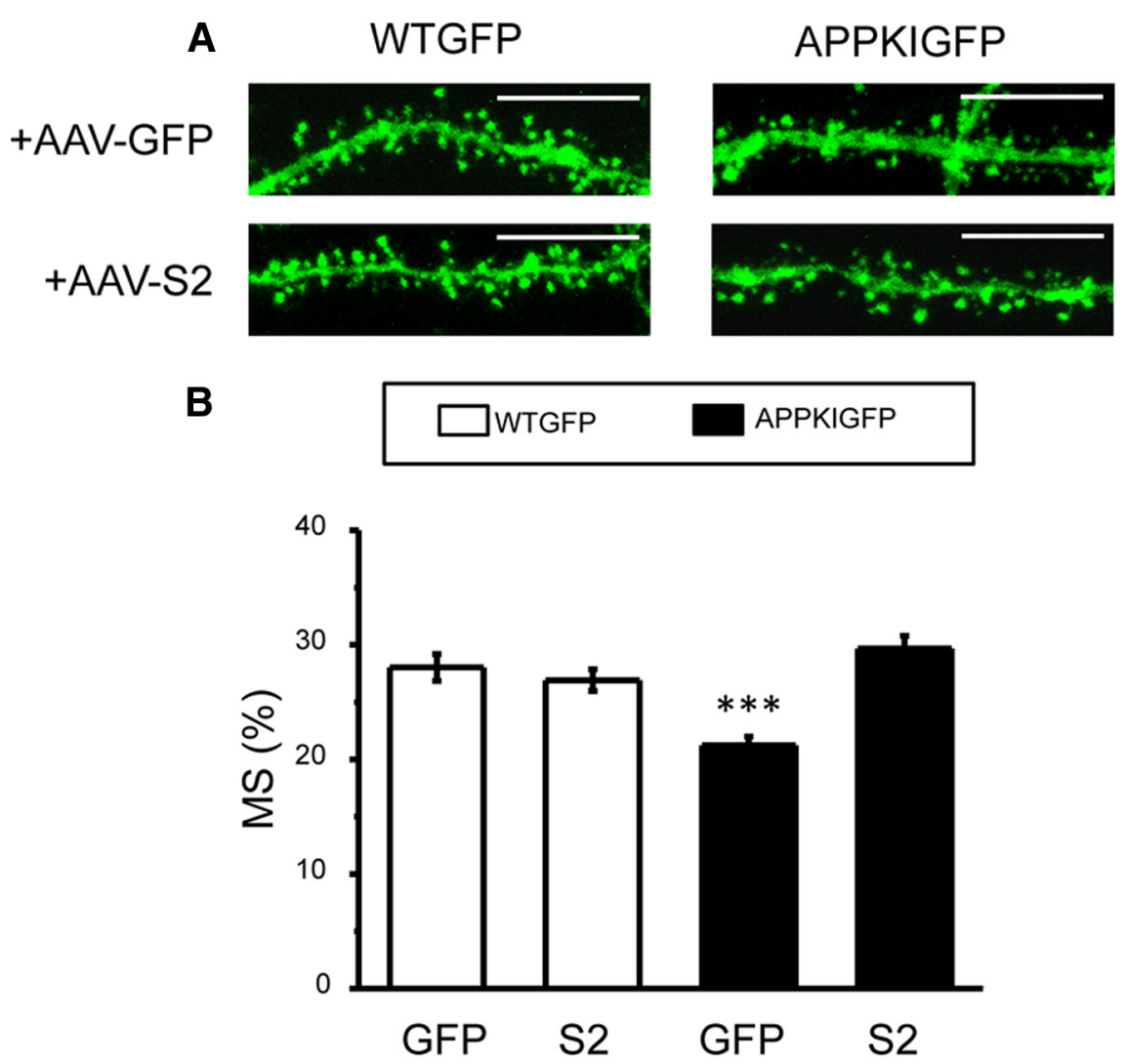

Figure 8. Overexpressing STIM2 rescues mushroom spine loss in APPKI mice in vivo. $A$, Confocal images of CA1 hippocampal neurons from 6-month-old WTGFP and APPKIGFP mice infected with AAV-GFP (+ AAV-GFP) and AAV-STIM2 (+ AAVS2) viruses. Scale bars, $10 \mu \mathrm{m}$. B, Fraction of mushroom spines (MS) in hippocampal slices from 6-month-old WTGFP and APPKIGFP mice infected with AAV-GFP (GFP) and AAV-STIM2 (S2) viruses. The average fraction of mushroom spines is shown as mean \pm SE ( $n \geq 23$ neurons, WTGFP + AAVGFP group from 3 mice, all other groups from 6 mice). ${ }^{* * *} p<0.001$. 

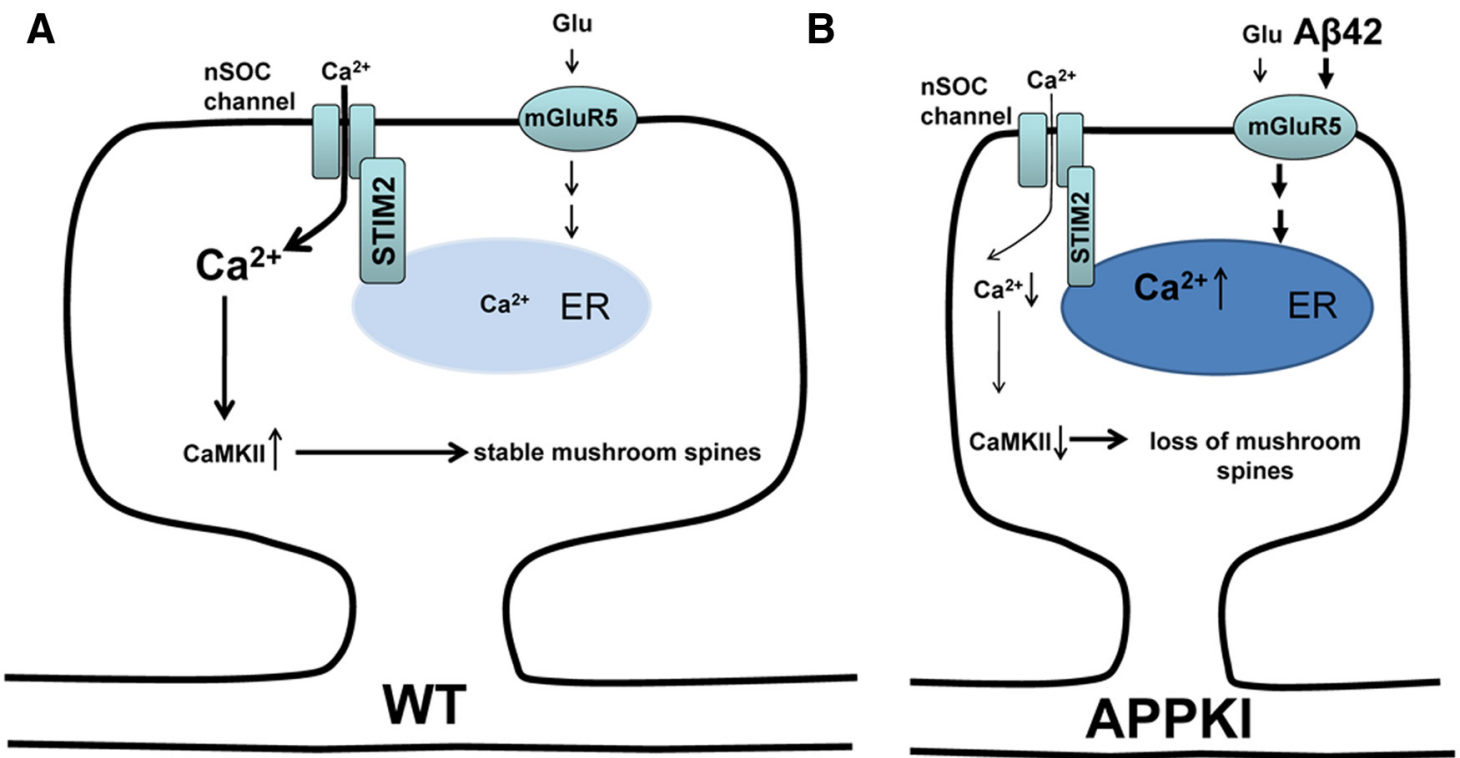

Figure 9. Synaptic $\mathrm{nSOC}$ and loss of mushroom spines in APPKI hippocampal neurons. A, Long-term maintenance of mushroom hippocampal spines in WT neurons depends on synaptic nSOC and activity of synaptic CaMKII (Sun et al., 2014). Synaptic nSOC is gated by STIM2 protein that is regulated by synaptic ER Ca ${ }^{2+}$ levels (Sun et al., 2014). Filling state of ER Ca ${ }^{2+}$ stores influenced by activity of synaptic mGluR5 receptors, which are activated by extracellular glutamate (Glu). $B$, Accumulation of $A \beta_{42}$ in extracellular media of APPKI neurons causes supernormal and continuous activation of synaptic mGluR5 receptors (Renner et al., 2010; Um et al., 2013). Resulting elevation of ER Ca ${ }^{2+}$ levels causes compensatory downregulation on STIM2 expression and synaptic nSOC. Reduced synaptic nSOC causes reduction in synaptic CaMKIl activity, leading to destabilization and loss of mushroom spines in APPKI neurons.

DLs to hippocampal cultures (Fig. 2A,B). Consistent with previous reports (Townsend et al., 2006; Jin et al., 2011), endogenous $\mathrm{A} \beta_{42}$ shows synaptotoxicity at a much lower concentration than synthetic $\mathrm{A} \beta_{42}$. Our estimate is that the concentration of $\mathrm{A} \beta_{42}$ is $\sim 0.36 \mathrm{nM}$ in APPKI culture medium at DIV15 when compared with $100 \mathrm{nM}$ to $1 \mu \mathrm{M} \mathrm{A} \beta_{42}$ concentration range used typically in experiments with synthetic ADDLs (Fig. $2 A$ ). The increased toxicity of these naturally derived $\mathrm{A} \beta$ assemblies has been explained by their "biologically active conformation" (Townsend et al., 2006) and also might be related to the heterogeneous character of natural aggregates or to unknown posttranslational modifications absent in synthetic $A \beta$ preparations (Benilova et al., 2012). It is also possible that, although overall $\mathrm{A} \beta_{42}$ concentration in APPKI culture medium is very low, the local $\mathrm{A} \beta_{42}$ concentration at synaptic locations is significantly higher than the global concentration in the medium.

A number of $\mathrm{Ca}^{2+}$ signaling pathways have been implicated in synaptotoxic effects of amyloid (Demuro et al., 2010). Our results suggested that $\mathrm{ER} \mathrm{Ca}^{2+}$ levels are elevated, somatic nSOC is inhibited, and synaptic nSOC is almost completely abolished in APPKI neurons (Fig. 3). Dysregulation of these $\mathrm{Ca}^{2+}$ signaling pathways was prevented by overnight incubation of APPKI neurons with the mGluR5 inhibitor MPEP (Fig. 5). Incubation with MPEP was also sufficient to rescue mushroom spine loss in APPKI neurons (Fig. 4). Other $\mathrm{Ca}^{2+}$ signaling inhibitors, such as the NR2B NMDAR inhibitor ifenprodil and the L-type VGCC inhibitor nifedipine, were significantly less effective than MPEP (Fig. 4). However, the novel specific mGluR5 antagonist PF-5212391 was equally effective in preventing mushroom spine loss in APPKI cultures (data not shown).

To explain these results, we propose that activation of mGluR5 in hippocampal neurons is important for refilling of ER $\mathrm{Ca}^{2+}$ stores (Fig. 9A). In cerebellar Purkinje cells, activation of mGluR1 triggers TRPC3-mediated $\mathrm{Ca}^{2+}$ influx and refilling of the $\mathrm{ER} \mathrm{Ca}^{2+}$ stores (Hartmann et al., 2014). It has been demonstrated that activation of mGluR $1 / 5$ is necessary for refilling of ER
$\mathrm{Ca}^{2+}$ stores in experiments with PC12 cells (Lv et al., 2014). We propose that a similar mechanism of mGluR5-dependent ER $\mathrm{Ca}^{2+}$ store refilling also exists in hippocampal neurons (Fig. 9A). In support of this hypothesis, overnight incubation with MPEP significantly reduced ER $\mathrm{Ca}^{2+}$ levels in WT neurons (Fig. 5A,B). In APPKI cultures, accumulation of $\mathrm{A} \beta_{42}$ in extracellular media caused persistent activation of mGluR5 (Renner et al., 2010; Um et al., 2013), resulting in supernormal ER $\mathrm{Ca}^{2+}$ levels (Fig. 9B). mGluR1/5-mediated refilling of ER $\mathrm{Ca}^{2+}$ stores depends primarily on STIM1 (Hartmann et al., 2014; Lv et al., 2014) and will not be affected by downregulation of STIM2 in APPKI neurons. We further reason that $\mathrm{ER} \mathrm{Ca}^{2+}$ overload in APPKI neurons leads to compensatory downregulation of STIM2 expression and synaptic nSOC, resulting in destabilization of mushroom spines in APPKI neurons (Fig. 9B). Indeed, overnight incubation with MPEP restored somatic and synaptic nSOC to WT levels in APPKI neurons (Fig. 5) and completely rescued mushroom spine loss in APPKI neurons (Fig. 4).

STIM2-nSOC-CaMKII pathway as a common pathway in AD pathogenesis and novel drug target

In a previous study, we demonstrated that downregulation of the synaptic STIM2-nSOC-CaMKII pathway appears to be responsible for mushroom spine loss in the PS1-M146V KI mouse model of AD (Sun et al., 2014). In the previous study, we reasoned that downregulation of the STIM2-nSOC pathway occurs in response to $\mathrm{ER} \mathrm{Ca}^{2+}$ overload resulting from the loss of ER $\mathrm{Ca}^{2+}$ leak function of presenilins (Sun et al., 2014). The present results suggest that similar dysregulation of synaptic $\mathrm{Ca}^{2+}$ signaling also occurs in APPKI neurons (Fig. 9B). In this case, ER Ca ${ }^{2+}$ overload occurs in response to $\mathrm{A} \beta_{42}$-induced superactivation of mGluR5 receptors (Fig. 9B). Similar to PS1-M146V KI neurons, this leads to compensatory downregulation of STIM2 and synaptic nSOC, reduction in activity of synaptic CaMKII, loss of PSD95, and loss of mushroom synaptic spines (Fig. 3). Also similar to studies with PS1-M146V KI neurons, overexpression of 
STIM2 was sufficient to rescue synaptic nSOC and restore levels of PSD95 and pCaMKII (Figs. 6, 7). Importantly, overexpression of STIM2 also resulted in rescue of mushroom synaptic spines in APPKI hippocampal neurons in vitro and in vivo (Figs. 7, 8). From these results, we conclude that downregulation of synaptic STIM2-nSOC-CaMKII is a common feature responsible for mushroom spine loss in presenilin and amyloid models of AD. Moreover, in a recent study, we demonstrated that downregulation of the synaptic STIM2-nSOC-CaMKII pathway occurs as a result of brain aging (Sun et al., 2014). We also obtained evidence that STIM2 protein is downregulated in sporadic AD human brains (Sun et al., 2014). Results presented in the present study and our previous one (Sun et al., 2014) suggest that activators of the STIM2-nSOC-CaMKII pathway may offer potential therapeutic benefit for $\mathrm{AD}$ and aging-related memory loss.

\section{References}

Anekonda TS, Quinn JF, Harris C, Frahler K, Wadsworth TL, Woltjer RL (2011) L-type voltage-gated calcium channel blockade with isradipine as a therapeutic strategy for Alzheimer's disease. Neurobiol Dis 41:62-70. CrossRef Medline

Benilova I, Karran E, De Strooper B (2012) The toxic Abeta oligomer and Alzheimer's disease: an emperor in need of clothes. Nat Neurosci 15:349357. CrossRef Medline

Bourne J, Harris KM (2007) Do thin spines learn to be mushroom spines that remember? Curr Opin Neurobiol 17:381-386. CrossRef Medline

Bourne JN, Harris KM (2008) Balancing structure and function at hippocampal dendritic spines. Annu Rev Neurosci 31:47-67. CrossRef Medline

De Felice FG, Velasco PT, Lambert MP, Viola K, Fernandez SJ, Ferreira ST, Klein WL (2007) Abeta oligomers induce neuronal oxidative stress through an $N$-methyl-D-aspartate receptor-dependent mechanism that is blocked by the Alzheimer drug memantine. J Biol Chem 282:1159011601. CrossRef Medline

Demuro A, Parker I, Stutzmann GE (2010) Calcium signaling and amyloid toxicity in Alzheimer disease. J Biol Chem 285:12463-12468. CrossRef Medline

Feng G, Mellor RH, Bernstein M, Keller-Peck C, Nguyen QT, Wallace M, Nerbonne JM, Lichtman JW, Sanes JR (2000) Imaging neuronal subsets in transgenic mice expressing multiple spectral variants of GFP. Neuron 28:41-51. CrossRef Medline

Ferreira IL, Bajouco LM, Mota SI, Auberson YP, Oliveira CR, Rego AC (2012) Amyloid beta peptide 1-42 disturbs intracellular calcium homeostasis through activation of GluN2B-containing $N$-methyl-D-aspartate receptors in cortical cultures. Cell Calcium 51:95-106. CrossRef Medline

Gandy S, Simon AJ, Steele JW, Lublin AL, Lah JJ, Walker LC, Levey AI, Krafft GA, Levy E, Checler F, Glabe C, Bilker WB, Abel T, Schmeidler J, Ehrlich ME (2010) Days to criterion as an indicator of toxicity associated with human Alzheimer amyloid-beta oligomers. Ann Neurol 68:220-230. CrossRef Medline

Hartmann J, Karl RM, Alexander RP, Adelsberger H, Brill MS, Rühlmann C, Ansel A, Sakimura K, Baba Y, Kurosaki T, Misgeld T, Konnerth A (2014) STIM1 controls neuronal $\mathrm{Ca}(2)(+)$ signaling, mGluR1-dependent synaptic transmission, and cerebellar motor behavior. Neuron 82:635-644. CrossRef Medline

Jin M, Shepardson N, Yang T, Chen G, Walsh D, Selkoe DJ (2011) Soluble amyloid beta-protein dimers isolated from Alzheimer cortex directly induce Tau hyperphosphorylation and neuritic degeneration. Proc Natl Acad Sci U S A 108:5819-5824. CrossRef Medline

Kasai H, Matsuzaki M, Noguchi J, Yasumatsu N, Nakahara H (2003) Structure-stability-function relationships of dendritic spines. Trends Neurosci 26:360-368. CrossRef Medline

Kim S, Rhim H (2011) Effects of amyloid-beta peptides on voltage-gated L-type $\mathrm{Ca}(\mathrm{V}) 1.2$ and $\mathrm{Ca}(\mathrm{V}) 1.3 \mathrm{Ca}(2+)$ channels. Mol Cells 32:289-294. CrossRef Medline

Koffie RM, Hyman BT, Spires-Jones TL (2011) Alzheimer's disease: synapses gone cold. Mol Neurodegener 6:63. CrossRef Medline

Li S, Jin M, Koeglsperger T, Shepardson NE, Shankar GM, Selkoe DJ (2011) Soluble Abeta oligomers inhibit long-term potentiation through a mech- anism involving excessive activation of extrasynaptic NR2B-containing NMDA receptors. J Neurosci 31:6627-6638. CrossRef Medline

Lv MM, Cheng YC, Xiao ZB, Sun MY, Ren PC, Sun XD (2014) Downregulation of Homerlb/c attenuates group I metabotropic glutamate receptors dependent $\mathrm{Ca}(2)(+)$ signaling through regulating endoplasmic reticulum $\mathrm{Ca}(2)(+)$ release in PC12 cells. Biochem Biophys Res Commun 450:1568-1574. CrossRef Medline

Mucke L, Selkoe DJ (2012) Neurotoxicity of amyloid beta-protein: synaptic and network dysfunction. Cold Spring Harb Perspect Med 2:a006338. CrossRef Medline

Popugaeva E, Bezprozvanny I (2013) Role of endoplasmic reticulum Ca2+ signaling in the pathogenesis of Alzheimer disease. Front Mol Neurosci 6:29. CrossRef Medline

Popugaeva E, Supnet C, Bezprozvanny I (2012) Presenilins, deranged calcium homeostasis, synaptic loss and dysfunction in Alzheimer's disease. Messenger 1:53-62. CrossRef

Price KA, Varghese M, Sowa A, Yuk F, Brautigam H, Ehrlich ME, Dickstein DL (2014) Altered synaptic structure in the hippocampus in a mouse model of Alzheimer's disease with soluble amyloid-beta oligomers and no plaque pathology. Mol Neurodegener 9:41. CrossRef Medline

Renner M, Lacor PN, Velasco PT, Xu J, Contractor A, Klein WL, Triller A (2010) Deleterious effects of amyloid beta oligomers acting as an extracellular scaffold for mGluR5. Neuron 66:739-754. CrossRef Medline

Rodriguez A, Ehlenberger DB, Dickstein DL, Hof PR, Wearne SL (2008) Automated three-dimensional detection and shape classification of dendritic spines from fluorescence microscopy images. PLoS One 3:e1997. CrossRef Medline

Saito T, Matsuba Y, Mihira N, Takano J, Nilsson P, Itohara S, Iwata N, Saido TC (2014) Single App knock-in mouse models of Alzheimer's disease. Nat Neurosci 17:661-663. CrossRef Medline

Selkoe DJ (2002) Alzheimer's disease is a synaptic failure. Science 298:789_ 791. CrossRef Medline

Sun S, Zhang H, Liu J, Popugaeva E, Xu NJ, Feske S, White CL 3rd, Bezprozvanny I (2014) Reduced synaptic STIM2 expression and impaired storeoperated calcium entry cause destabilization of mature spines in mutant presenilin mice. Neuron 82:79-93. Medline

Sun X, Beglopoulos V, Mattson MP, Shen J (2005) Hippocampal spatial memory impairments caused by the familial Alzheimer's disease-linked presenilin 1 M146V mutation. Neurodegener Dis 2:6-15. CrossRef Medline

Tackenberg C, Brandt R (2009) Divergent pathways mediate spine alterations and cell death induced by amyloid- $\beta$, wild-type tau, and R406W tau. J Neurosci 29:14439-14450. CrossRef Medline

Tackenberg C, Ghori A, Brandt R (2009) Thin, stubby or mushroom: spine pathology in Alzheimer's disease. Curr Alzheimer Res 6:261-268. CrossRef Medline

Tian L, Hires SA, Mao T, Huber D, Chiappe ME, Chalasani SH, Petreanu L, Akerboom J, McKinney SA, Schreiter ER, Bargmann CI, Jayaraman V, Svoboda K, Looger LL (2009) Imaging neural activity in worms, flies and mice with improved GCaMP calcium indicators. Nat Methods 6:875881. CrossRef Medline

Townsend M, Shankar GM, Mehta T, Walsh DM, Selkoe DJ (2006) Effects of secreted oligomers of amyloid beta-protein on hippocampal synaptic plasticity: a potent role for trimers. J Physiol 572:477-492. CrossRef Medline

Tu S, Okamoto S, Lipton SA, Xu H (2014) Oligomeric Abeta-induced synaptic dysfunction in Alzheimer's disease. Mol Neurodegener 9:48. CrossRef Medline

Um JW, Kaufman AC, Kostylev M, Heiss JK, Stagi M, Takahashi H, Kerrisk ME, Vortmeyer A, Wisniewski T, Koleske AJ, Gunther EC, Nygaard HB, Strittmatter SM (2013) Metabotropic glutamate receptor 5 is a coreceptor for Alzheimer abeta oligomer bound to cellular prion protein. Neuron 79:887-902. CrossRef Medline

Wang R, Dineley KT, Sweatt JD, Zheng H (2004) Presenilin 1 familial Alzheimer's disease mutation leads to defective associative learning and impaired adult neurogenesis. Neuroscience 126:305-312. CrossRef Medline

Wang Y, Mattson MP (2014) L-type Ca2 + currents at CA1 synapses, but not $\mathrm{CA} 3$ or dentate granule neuron synapses, are increased in 3xTgAD mice in an age-dependent manner. Neurobiol Aging 35:88-95. CrossRef Medline

Zhang H, Sun S, Herreman A, De Strooper B, Bezprozvanny I (2010) Role of presenilins in neuronal calcium homeostasis. J Neurosci 30:8566-8580. CrossRef Medline

Zhang H, Liu J, Sun S, Pchitskaya E, Popugaeva E, Bezprozvanny I (2015) Calcium signaling, excitability, and synaptic plasticity defects in a mouse model of Alzheimer's disease. J Alzheimers Dis 45:561-580. CrossRef Medline 